\title{
The Einstein relation in quantum wires of III-V, ternary, and quaternary materials in the presence of light waves: Simplified theory, relative comparison, and suggestion for experimental determination
}

\author{
K. P. Ghatak, ${ }^{1, a)}$ S. Bhattacharya,${ }^{2}$ S. Bhowmik, ${ }^{3}$ R. Benedictus, ${ }^{3}$ and S. Choudhury ${ }^{4}$ \\ ${ }^{1}$ Department of Electronic Science, The University of Calcutta, 92, Acharyya Prafulla Road, \\ Kolkata 700 009, India \\ ${ }^{2}$ Nanoscale Device Research Laboratory, Centre for Electronics Design and Technology, Indian Institute of \\ Science, Bangalore 560 012, India \\ ${ }^{3}$ Faculty of Aerospace Engineering, Delft University of Technology, Kluyverweg 1, 2629 HS Delft, The \\ Netherlands \\ ${ }^{4}$ Department of Electronics and Communication Engineering, Sikkim Manipal Institute of Technology, \\ Majitar, Rangpo, East Sikkim 737 132, India
}

(Received 16 November 2007; accepted 2 March 2008; published online 8 May 2008)

\begin{abstract}
We study the Einstein relation for the diffusivity to mobility ratio (DMR) in quantum wires (QWs) of III-V, ternary, and quaternary materials in the presence of light waves, whose unperturbed energy band structures are defined by the three band model of Kane. It has been found, taking $n$-InAs, $n$-InSb, $n-\mathrm{Hg}_{1-x} \mathrm{Cd}_{x} \mathrm{Te}, n-\mathrm{In}_{1-x} \mathrm{Ga}_{x} \mathrm{As}_{y} \mathrm{P}_{1-y}$ lattice matched to $\mathrm{InP}$ as examples, that the respective DMRs exhibit decreasing quantum step dependence with the increasing film thickness, decreasing electron statistics, increasing light intensity and wavelength, with different numerical values. The nature of the variations is totally band structure dependent and is influenced by the presence of the different energy band constants. The strong dependence of the DMR on both the light intensity and the wavelength reflects the direct signature of the light waves which is in contrast as compared to the corresponding QWs of the said materials in the absence of photoexcitation. The classical equation of the DMR in the absence of any field has been obtained as a special case of the present analysis under certain limiting conditions and this is the indirect test of the generalized formalism. We have suggested an experimental method of determining the DMR in QWs of degenerate materials having arbitrary dispersion laws and our results find six applications in the field of quantum effect devices. (C) 2008 American Institute of Physics. [DOI: 10.1063/1.2913515]
\end{abstract}

\section{INTRODUCTION}

The Einstein relation for the diffusivity $(D)$ to mobility $(\mu)$ ratio $(\mathrm{DMR})$ of the carriers in semiconductor devices is known to be very useful, ${ }^{1}$ since the diffusion constant (a quantity often used in device analysis but whose exact experimental determination is rather difficult) can be obtained from this ratio by knowing the experimental values of the mobility. In addition, it is more accurate than any of the individual relation for the diffusivity or the mobility, which are the two widely used quantities of carrier transport of modern nanostructured materials and devices. The classical value of the DMR is equal to $\left(k_{B} T / e\right)$, where $k_{B}, T$, and $e$ are the Boltzmann constant, temperature, and the magnitude of the carrier charge, respectively, which represents the wellknown Einstein relation and is valid for both types of carriers. In this conventional form, it appears that, the DMR increases linearly with $T$ being independent of the carrier concentration. This relation holds only under the condition of carrier nondegeneracy, although its validity has been erroneously suggested for degenerate materials. ${ }^{2}$ The performance of the electron devices at the device terminals and the speed of operation of modern switching transistors are significantly influence by the degree of carrier degeneracy present in these

${ }^{a)}$ Electronic mail: kamakhyaghatak@yahoo.co.in. devices, ${ }^{3}$ and the simplest way of analyzing them under degenerate condition is to use the appropriate Einstein relation to express the performance of the devices at the device terminals and the switching speed in terms of the carrier concentration. ${ }^{3}$

It is well-known from the fundamental work of Landsberg ${ }^{4}$ that the Einstein relation for electronic materials having degenerate electron concentration is essentially determined by their respective energy band structures. It has, therefore, different values in different materials and varies with the doping, with the magnitude of the reciprocal quantizing magnetic field under magnetic quantization, with the quantizing electric field as in inversion layers, with the nanothickness as in quantum wells (QWs) and QW wires, and with superlattice period as in the quantum confined superlattices of small gap semiconductors with graded interfaces having various carrier energy spectra. The nature of these variations has been extensively investigated in literature ${ }^{4-34}$ and some of the significant features, which have emerged from these studies, are the following:

(a) The ratio increases monotonically with the increasing electron concentration.

(b) The ratio increases with doping in heavily doped materials in the presence of band tails.

(c) The nature of variations is significantly influenced by 
the energy band constants of various materials having different band structures.

(d) The ratio oscillates with inverse quantizing magnetic field due to Shubnikov-de Haas effect.

(e) The ratio increases with the magnitude of the quantizing electric field in inversion layers

(f) The ratio exhibits composite oscillations with significantly different values in superlattices and also in quantum confined semiconductors.

The above information has been obtained through theoretical analyses and no experimental results are available in literature in support of the predictions for QWs under photoexcitation. In this paper, the experimental method of determination of the DMR in QWs for materials having arbitrary band structures has been suggested and we have studied the one-dimensional (1D) DMR for QWs of III-V, ternary, and quaternary compounds in the presence of light waves.

With the advent of molecular beam epitaxy, fine line lithography, and other experimental techniques, lowdimensional structures having quantum confinement of one, two, and three dimensions (such as QWs, wires, and dots) have attracted much attention not only for their potential in uncovering new phenomena in nanoscience but also for their interesting applications in nanotechnology. ${ }^{35-37}$ In QWs, the restriction of the motion of the carriers in the two directions may be viewed as carrier confinement by two infinitely deep 1D square potential wells, leading to quantization (known as quantum size effect) of the wave vectors along the two directions, allowing $1 \mathrm{D}$ electron transport representing new physical features not exhibited in bulk semiconductors. The low-dimensional heterostructures based on various materials are widely investigated because of the enhancement of carrier mobility. An enormous range of important applications for nanoscience in the quantum regime together with a rapid increase in computing power have generated much interest in the analysis of nanostructured devices for investigating their properties. ${ }^{38-41}$ Examples of such new applications are based on the different transport properties of ballistic charge carriers which include quantum wires, ${ }^{42-46}$ quantum resistors, ${ }^{47}$ resonant tunneling diodes and band filters, ${ }^{48,49}$ quantum switches, ${ }^{50}$ quantum sensors, ${ }^{51-53}$ quantum logic gates, ${ }^{54,55}$ quantum transistors and subtuners, ${ }^{56-58}$ heterojunction fieldeffect transistors, ${ }^{59}$ high-speed digital networks, ${ }^{60}$ highfrequency microwave circuits, ${ }^{61}$ optical modulators, ${ }^{62}$ optical switching systems, ${ }^{63}$ and other devices. The III-V materials are being increasingly used in integrated optoelectronics, ${ }^{64}$ passive filter devices, ${ }^{65}$ distributed feedback lasers and Bragg reflectors. $^{66}$ We have used $n-\mathrm{Hg}_{1-x} \mathrm{Cd}_{x} \mathrm{Te}$ and $n$ - $\mathrm{In}_{1-x} \mathrm{Ga}_{x} \mathrm{As}_{y} \mathrm{P}_{1-y}$ lattice matched to InP as examples of ternary and quaternary compounds, respectively. The $n-\mathrm{Hg}_{1-x} \mathrm{Cd}_{x} \mathrm{Te}$ is a classic narrow-gap compound and is an important optoelectronic material because its band gap can be varied to cover a spectral range from 0.8 to over $30 \mu \mathrm{m}$ by adjusting the alloy composition. ${ }^{67}$ The $n-\mathrm{Hg}_{1-x} \mathrm{Cd}_{x} \mathrm{Te}$ finds extensive applications in infrared detector materials ${ }^{68}$ and photovoltaic detector arrays ${ }^{69}$ in the $8-12 \mu \mathrm{m}$ wave

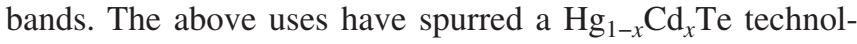
ogy for the production of high mobility single crystals, with specially prepared surface layers which is also ideally suitable for narrow subband physics because the relevant material constants are within the experimental reach. ${ }^{70}$ Moreover, the quaternary compounds also find extensive applications in optoelectronics, high electron mobility transistors, visible heterostructure compounds lasers, infrared light emitting diodes, lasers for fiber optic systems, ${ }^{71}$ tandem solar cells, ${ }^{72}$ avalanche photodetectors, ${ }^{73}$ long wavelength light sources, detectors in optical fiber communications, ${ }^{74}$ and new types of optical devices that are also made from the quaternary systems. $^{75}$

In Sec. II A, we have formulated the dispersion relation of the conduction electrons in the presence of light waves of III-V, ternary, and quaternary materials, whose energy band structures in the absence of photoexcitation are defined by the three and two band models of Kane together with the parabolic energy bands. The expressions for the electron concentration and DMR for QWs of the aforementioned materials in the presence and absence of photoexcitation have been formulated in Secs. II B and II C, respectively. In Secs. II D and II E, we have formulated the DMR both in the presence and absence of light waves for bulk materials of the aforementioned compounds, respectively. In Sec. II F, we have suggested an experimental method of determining the DMR in nanomaterials having arbitrary carrier energy spectra in the present case. In Sec. II G, we have suggested six specific applications of the results of this paper in the fields of quantum effect devices. The DMR has been numerically investigated by using $n$-InAs and $n$-InSb as examples of III-V compounds, $n-\mathrm{Hg}_{1-x} \mathrm{Cd}_{x} \mathrm{Te}$ as an example of ternary compounds, and $n-\mathrm{In}_{1-x} \mathrm{Ga}_{x} \mathrm{As}_{y} \mathrm{P}_{1-y}$ lattice matched to InP as an example of quaternary materials in accordance with the three and the two band models of Kane together with parabolic energy bands, respectively, for the purpose of relative comparison both in the presence and absence of light waves.

\section{THEORETICAL BACKGROUND}

\section{A. Formulation of the dispersion relation of the conduction electrons of III-V, ternary, and quaternary materials in the presence of light waves}

The Hamiltonian $(\hat{H})$ of an electron in the presence of light wave characterized by the vector potential $\vec{A}$ can be written as the following: ${ }^{76}$

$$
\hat{H}=\left[|(\hat{p}+|e| \vec{A})|^{2} / 2 m\right]+V(\vec{r}),
$$

in which $\hat{p}$ is the momentum operator, $V(\vec{r})$ is the crystal potential, and $m$ is the free electron mass. Equation (1) can be expressed as

$$
\hat{H}=\hat{H}_{0}+\hat{H}^{\prime}
$$

where $\hat{H}_{0}=\hat{p}^{2} / 2 m+V(\vec{r})$ and

$$
\hat{H}^{\prime}=\frac{|e|}{2 m} \vec{A} \cdot \hat{p} .
$$

The perturbed Hamiltonian $\hat{H}^{\prime}$ can be written as 


$$
\hat{H}^{\prime}=\left(\frac{-i \hbar|e|}{2 m}\right)(\vec{A} \cdot \nabla),
$$

where $i=\sqrt{-1}$ and $\hat{p}=-i \hbar \nabla$. The vector potential $(\vec{A})$ of the monochromatic light of plane wave can be expressed as

$$
\vec{A}=A_{0} \vec{\varepsilon}_{s} \cos \left(\vec{s}_{0} \cdot \vec{r}-\omega t\right),
$$

where $A_{0}$ is the amplitude of the light wave, $\vec{\varepsilon}_{s}$ is the polarization vector, $\vec{s}_{0}$ is the momentum vector of the incident photon, $\vec{r}$ is the position vector, $\omega$ is the angular frequency of light wave, and $t$ is the time scale. The matrix element of $\hat{H}^{\prime}{ }_{n l}$ between initial state $\psi_{l}(\vec{q}, \vec{r})$ and final state $\psi_{n}(\vec{k}, \vec{r})$ in different bands can be written as

$$
\hat{H}_{n l}^{\prime}=\frac{|e|}{2 m}\langle n \vec{k}|\vec{A} \cdot \hat{p}| l \vec{q}\rangle .
$$

By using Eqs. (4) and (5), we can rewrite Eq. (6) as

$$
\begin{aligned}
\hat{H}_{n l}^{\prime}= & \left(\frac{-i \hbar|e| A_{0}}{4 m}\right) \vec{\varepsilon}_{s} \cdot\left[\left(\left\langle n \vec{k}\left|e^{\left(i \vec{s}_{0} \cdot \vec{r}\right)} \nabla\right| l \vec{q}\right\rangle e^{-i \omega t}\right)\right. \\
& \left.+\left(\left\langle n \vec{k}\left|e^{\left(-i \overrightarrow{s_{0}} \cdot \vec{r}\right)} \nabla\right| l \vec{q}\right\rangle e^{i \omega t}\right)\right] .
\end{aligned}
$$

The first matrix element of Eq. (7) can be written as

$$
\begin{aligned}
\left\langle n \vec{k}\left|e^{\left(i \vec{s}_{0} \cdot \vec{r}\right)} \nabla\right| l \vec{q}\right\rangle= & \int e^{\left[i\left(\vec{q}+\overrightarrow{s_{0}}-\vec{k}\right) \cdot \vec{r}\right]} i \vec{q} u_{n}^{*}(\vec{k}, \vec{r}) u_{l}(\vec{q}, \vec{r}) d^{3} r \\
& +\int e^{\left[i\left(\vec{q}+\overrightarrow{s_{0}}-\vec{k}\right) \cdot \vec{r}\right]} u_{n}^{*}(\vec{k}, \vec{r}) \nabla u_{l}(\vec{q}, \vec{r}) d^{3} r .
\end{aligned}
$$

The functions $u_{n}^{*} u_{l}$ and $u_{n}^{*} \nabla u_{l}$ are periodic. The integral over all space can be separated into a sum over unit cells times an integral over a single unit cell. It is assumed that the wavelength of the electromagnetic wave is sufficiently large so that if $\vec{k}$ and $\vec{q}$ are within the Brillouin zone, $\left(\vec{q}+\vec{s}_{0}-\vec{k}\right)$ is not a reciprocal lattice vector.

Therefore, we can write Eq. (8) as

$$
\begin{aligned}
\langle n \vec{k}| & e^{\left(i \vec{s}_{0} \cdot \vec{r}\right)} \nabla|l \vec{q}\rangle \\
= & {\left[\frac{(2 \pi)^{3}}{\Omega}\right]\left[i \bar{q} \delta\left(\vec{q}+\vec{s}_{0}-\vec{k}\right) \delta_{n l}+\delta\left(\vec{q}+\vec{s}_{0}-\vec{k}\right)\right.} \\
& \left.\quad \times \int_{\text {cell }} u_{n}^{*}(\vec{k}, \vec{r}) \nabla u_{l}(\vec{q}, \vec{r}) d^{3} r\right] \\
= & {\left[\frac{(2 \pi)^{3}}{\Omega}\right]\left[\delta\left(\vec{q}+\vec{s}_{0}-\vec{k}\right) \int_{\text {cell }} u_{n}^{*}(\vec{k}, \vec{r}) \nabla u_{l}(\vec{q}, \vec{r}) d^{3} r\right], }
\end{aligned}
$$

where $\Omega$ is the volume of the unit cell and $\int u_{n}^{*}(\vec{k}, \vec{r}) u_{l}(\vec{q}, \vec{r}) d^{3} r=\delta(\vec{q}-\vec{k}) \delta_{n l}=0$, since $n \neq l$. The delta function expresses the conservation of wave vector in the absorption of light wave and $\vec{s}_{0}$ is small compared to the dimension of a typical Brillouin zone, and we set $\vec{q}=\vec{k}$. From Eqs. (8) and (9), we can write

$$
\hat{H}_{n l}^{\prime}=\frac{|e| A_{0}}{2 m} \vec{\varepsilon}_{s} \cdot \hat{p}_{n l}(\vec{k}) \delta(\vec{q}-\vec{k}) \cos (\omega t),
$$

where $\hat{p}_{n l}(\vec{k})=-i \hbar \int u_{n}^{*} \nabla u_{l} d^{3} r=\int u_{n}^{*}(\vec{k}, \vec{r}) \hat{p} u_{l}(\vec{k}, \vec{r}) d^{3} r \quad$ Therefore, we can write

$$
\hat{H}_{n l}^{\prime}=\frac{|e| A_{0}}{2 m} \vec{\varepsilon} \cdot \hat{p}_{n l}(\vec{k}),
$$

where $\vec{\varepsilon}=\vec{\varepsilon}_{s} \cos \omega t$.

When a photon interacts with a semiconductor, the carriers (i.e., electrons) are generated in the bands which are followed by the interband transitions. For example, when the carriers are generated in the valence band, the carriers then make interband transition to the conduction band. The transition of the electrons within the same band, i.e., $\hat{H}^{\prime}{ }_{n n}$ $=\left\langle n \vec{k}\left|\hat{H}^{\prime}\right| n \vec{k}\right\rangle$ is neglected. Because, in such a case, i.e., when the carriers are generated within the same bands by photons, they are lost by recombination within the aforementioned band resulting zero carriers.

Therefore,

$$
\left\langle n \vec{k}\left|\hat{H}^{\prime}\right| n \vec{k}\right\rangle=0 .
$$

With $n=c$ stands for conduction band and $l=v$ stands for valance band, the energy equation for the conduction electron can approximately be expressed as

$$
\gamma(E)=\left(\frac{\hbar^{2} k^{2}}{2 m^{*}}\right)+\frac{\left(\frac{|e| A_{0}}{2 m}\right)^{2}\left\langle\left|\vec{\varepsilon} \cdot \hat{p}_{c v}(\vec{k})\right|^{2}\right\rangle_{\mathrm{av}}}{E_{c}(\vec{k})-E_{v}(\vec{k})},
$$

where $\gamma(E) \equiv E(a E+1)(b E+1) /(c E+1), a \equiv 1 / E_{g_{0}}, E_{g_{0}}$ is the unperturbed band gap, $b \equiv 1 /\left(E_{g_{0}}+\Delta\right), \Delta$ is the spin-orbit splitting constant in the absence of any field, $c \equiv 1 /\left(E_{g_{0}}\right.$ $+2 \Delta / 3)$, and $\left\langle\left|\vec{\varepsilon} \cdot \hat{p}_{c v}(\vec{k})\right|^{2}\right\rangle_{\text {av }}$ represents the average of the square of the optical matrix element (OME). For the three band model of Kane, we can write

$$
\xi_{1 k}=E_{c}(\vec{k})-E_{v}(\vec{k})=\left(E_{g_{0}}^{2}+E_{g_{0}} \hbar^{2} k^{2} / m_{r}\right)^{1 / 2},
$$

where $m_{r}$ is the reduced mass and is given by $m_{r}^{-1}=\left(m^{*}\right)^{-1}$ $+m_{v}^{-1}$, and $m_{v}$ is the effective mass of the heavy hole at the top of the valance band in the absence of any field.

The doubly degenerate wave functions $u_{1}(\vec{k}, \vec{r})$ and $u_{2}(\vec{k}, \vec{r})$ can be expressed as ${ }^{21}$

$$
u_{1}(\vec{k}, \vec{r})=a_{k+}\left[(i s) \downarrow^{\prime}\right]+b_{k+}\left[\frac{X^{\prime}-i Y^{\prime}}{\sqrt{2}} \uparrow^{\prime}\right]+c_{k+}\left[Z^{\prime} \downarrow_{1}\right]
$$

and

$$
u_{2}(\vec{k}, \vec{r})=a_{k-}\left[(i s) \uparrow^{\prime}\right]-b_{k-}\left[\frac{X^{\prime}+i Y^{\prime}}{\sqrt{2}} \downarrow^{\prime}\right]+c_{k-}\left[Z^{\prime} \uparrow^{\prime}\right],
$$

where $s$ is the $s$-type atomic orbital in both unprimed and primed coordinates, $\downarrow^{\prime}$ indicates the spin-down function in the primed coordinates,

$$
\left.a_{k \pm} \equiv \beta\left[E_{g_{0}}-\left(\gamma_{0 k \pm}\right)^{2}\left(E_{g_{0}}-\delta^{\prime}\right)\right]^{1 / 2}\left(E_{g_{0}}+\delta^{\prime}\right)^{-1 / 2}\right],
$$




$$
\begin{aligned}
& \beta \equiv\left\{\left[6\left(E_{g_{0}}+2 \Delta / 3\right)\left(E_{g_{0}}+\Delta\right)\right] / \chi\right\}^{1 / 2}, \\
& \chi \equiv\left(6 E_{g_{0}}^{2}+9 E_{g_{0}} \Delta+4 \Delta^{2}\right), \\
& \gamma_{0 k \pm} \equiv\left[\frac{\left(\xi_{1 k} \mp E_{g_{0}}\right)}{2\left(\xi_{1 k}+\delta^{\prime}\right)}\right]^{1 / 2}, \\
& \xi_{1 k} \equiv E_{c}(\vec{k})-E_{v}(\vec{k})=E_{g_{0}}\left[1+2\left(1+\frac{m_{c}}{m_{v}}\right) \frac{\gamma(E)}{E_{g_{0}}}\right]^{1 / 2},
\end{aligned}
$$

$\delta^{\prime} \equiv\left(E_{g_{0}}^{2} \Delta\right)(\chi)^{-1} X^{\prime}, Y^{\prime}$, and $Z^{\prime}$ are the $p$-type atomic orbitals in the primed coordinates, $\uparrow^{\prime}$ indicates the spin-up function in the primed coordinates, $b_{k \pm} \equiv \rho \gamma_{0 k \pm}, \rho \equiv\left(4 \Delta^{2} / 3 \chi\right)^{1 / 2}$, $c_{k \pm} \equiv t \gamma_{0 k \pm}$, and

$$
t \equiv\left[6\left(E_{g_{0}}+2 \Delta / 3\right)^{2} / \chi\right]^{1 / 2} .
$$

We can, therefore, write the expression for the OME as

$$
\mathrm{OME}=\hat{p}_{c v}(\vec{k})=\left\langle u_{1}(\vec{k}, \vec{r})|\hat{p}| u_{2}(\vec{k}, \vec{r})\right\rangle .
$$

Since the photon vector has no interaction in the same band for the study of interband optical transition, we can, therefore, write

$$
\langle S|\hat{p}| S\rangle=\langle X|\hat{p}| X\rangle=\langle Y|\hat{p}| Y\rangle=\langle Z|\hat{p}| Z\rangle=0
$$

and

$$
\langle X|\hat{p}| Y\rangle=\langle Y|\hat{p}| Z\rangle=\langle Z|\hat{p}| X\rangle=0 .
$$

There are finite interactions between the conduction band (CB) and the valance band (VB), and we can obtain

$$
\begin{aligned}
& \langle S|\hat{P}| X\rangle=\hat{i} \cdot \hat{P}=\hat{i} \cdot \hat{P}_{x}, \\
& \left\langle S|\hat{P}|_{Y\rangle}=\hat{j} \cdot \hat{P}=\hat{j} \cdot \hat{P}_{y},\right. \\
& \left\langle S|\hat{P}|_{Z\rangle}=\hat{k} \cdot \hat{P}=\hat{k} \cdot \hat{P}_{z},\right.
\end{aligned}
$$

where $\hat{i}, \hat{j}$, and $\hat{k}$ are the unit vectors along the $x, y$, and $z$ axes, respectively.

It is well-known that

$$
\begin{aligned}
{\left[\begin{array}{l}
\uparrow^{\prime} \\
\downarrow^{\prime}
\end{array}\right]=} & {\left[\begin{array}{cc}
e^{-i \phi / 2} \cos (\theta / 2) & e^{i \phi / 2} \sin (\theta / 2) \\
-e^{-i \phi / 2} \sin (\theta / 2) & e^{i \phi / 2} \cos (\theta / 2)
\end{array}\right] } \\
& \times\left[\begin{array}{l}
\uparrow \\
\downarrow
\end{array}\right] \text { and }\left[\begin{array}{c}
X^{\prime} \\
Y^{\prime} \\
Z^{\prime}
\end{array}\right] \\
= & {\left[\begin{array}{ccc}
\cos \theta \cos \phi & \cos \theta \sin \phi & -\sin \theta \\
-\sin \phi & \cos \phi & 0 \\
\sin \theta \cos \phi & \sin \theta \sin \phi & \cos \theta
\end{array}\right]\left[\begin{array}{l}
X \\
Y \\
Z
\end{array}\right] . }
\end{aligned}
$$

Besides, the spin vector can be written as

$$
\vec{S}=\frac{\hbar}{2} \vec{\sigma},
$$

where

$$
\sigma_{x}=\left[\begin{array}{ll}
0 & 1 \\
1 & 0
\end{array}\right], \quad \sigma_{y}=\left[\begin{array}{cc}
0 & -i \\
i & 0
\end{array}\right] \quad \text { and } \sigma_{z}=\left[\begin{array}{cc}
1 & 0 \\
0 & -1
\end{array}\right] .
$$

From above, we can write

$$
\begin{aligned}
\hat{p}_{C V}(\vec{k})= & \left\langle u_{1}(\vec{k}, \vec{r})|\hat{P}| u_{2}(\vec{k}, \vec{r})\right\rangle \\
= & \left\langle\left\{a_{k_{+}}\left[(i S) \downarrow^{\prime}\right]+b_{k_{+}}\left[\left(\frac{X^{\prime}-i Y^{\prime}}{\sqrt{2}}\right) \uparrow^{\prime}\right]+c_{k_{+}}\left[Z^{\prime} \downarrow^{\prime}\right]\right\}\right. \\
& \times|\hat{P}|\left\{a_{k_{-}}\left[(i S) \uparrow^{\prime}\right]-b_{k_{-}}\left[\left(\frac{X^{\prime}+i Y^{\prime}}{\sqrt{2}}\right) \downarrow^{\prime}\right.\right. \\
& \left.\left.\left.+c_{k_{-}}\left(Z^{\prime} \uparrow^{\prime}\right)\right]\right\}\right\rangle .
\end{aligned}
$$

By using above relations, we get

$$
\begin{aligned}
\hat{p}_{C V}(\vec{k})= & \left\langle u_{1}(\vec{k}, \vec{r})|\hat{P}| u_{2}(\vec{k}, \vec{r})\right\rangle \\
= & \frac{b_{k_{+}} a_{k_{-}}}{\sqrt{2}}\left[\left\langle\left(X^{\prime}-i Y^{\prime}\right)|\hat{P}| i S\right\rangle\left\langle\uparrow^{\prime} \mid \uparrow^{\prime}\right\rangle\right] \\
& +c_{k_{+}} a_{k_{-}}\left[\left\langle Z^{\prime}|\hat{P}| i S\right\rangle\left\langle\downarrow^{\prime} \mid \uparrow^{\prime}\right\rangle\right]-\frac{a_{k_{+}} b_{k_{-}}}{\sqrt{2}}\left[\left\langlei S | \hat { P } | \left(X^{\prime}\right.\right.\right. \\
& \left.\left.\left.+i Y^{\prime}\right)\right\rangle\left\langle\downarrow^{\prime} \mid \downarrow^{\prime}\right\rangle\right]+a_{k_{+}} c_{k_{-}}\left(\left\langle i S|\hat{P}| Z^{\prime}\right\rangle\left\langle\downarrow^{\prime} \mid \uparrow^{\prime}\right\rangle\right) .
\end{aligned}
$$

From Eq. (18), we can write

$$
\begin{aligned}
\left\langle\left(X^{\prime}-i Y^{\prime}\right)|\hat{P}| i S\right\rangle & =\left\langle\left(X^{\prime}\right)|\hat{P}| i S\right\rangle-\left\langle\left(i Y^{\prime}\right)|\hat{P}| i S\right\rangle \\
& =i \int u_{X^{\prime}}^{*} \hat{P} S-\int-i u_{Y^{\prime}}^{*} \hat{P} i u_{X} \\
& =i\left\langle X^{\prime}|\hat{P}| S\right\rangle-\left\langle Y^{\prime}|\hat{P}| S\right\rangle .
\end{aligned}
$$

From the above relations, for $X^{\prime}, X^{\prime}$, and $X^{\prime}$, we get

$$
\left|X^{\prime}\right\rangle=\cos \theta \cos \phi|X\rangle+\cos \theta \sin \phi|Y\rangle-\sin \theta|Z\rangle .
$$

Thus,

$$
\begin{aligned}
\left\langle X^{\prime}|\hat{P}| S\right\rangle= & \cos \theta \cos \phi\langle X|\hat{P}| S\rangle+\cos \theta \sin \phi\langle Y|\hat{P}| S\rangle \\
& -\sin \theta\langle Z|\hat{P}| S\rangle=\hat{P} \hat{r}_{1},
\end{aligned}
$$

where

$$
\begin{aligned}
& \hat{r}_{1}=\hat{i} \cos \theta \cos \phi+\hat{j} \cos \theta \sin \phi-\hat{k} \sin \theta, \\
& \left|Y^{\prime}\right\rangle=-\sin \phi|X\rangle+\cos \phi|Y\rangle+0|Z\rangle .
\end{aligned}
$$

Thus,

$$
\begin{aligned}
\left\langle Y^{\prime}|\hat{P}| S\right\rangle & =-\sin \phi\langle X|\hat{P}| S\rangle+\cos \phi\langle Y|\hat{P}| S\rangle+0\langle Z|\hat{P}| S\rangle \\
& =\hat{P} \hat{r}_{2},
\end{aligned}
$$

where

$$
\hat{r}_{2}=-\hat{i} \sin \phi+\hat{j} \cos \phi,
$$

so that

$$
\left\langle\left(X^{\prime}-i Y^{\prime}\right)|\hat{P}| S\right\rangle=\hat{P}\left(i \hat{r}_{1}-\hat{r}_{2}\right) .
$$

Thus, 


$$
\frac{a_{k_{-}} b_{k_{+}}}{\sqrt{2}}\left\langle\left(X^{\prime}-i Y^{\prime}\right)|\hat{P}| S\right\rangle\left\langle\uparrow^{\prime} \mid \uparrow^{\prime}\right\rangle=\frac{a_{k_{-}} b_{k_{+}}}{\sqrt{2}} \hat{P}\left(i \hat{r}_{1}-\hat{r}_{2}\right)\left\langle\uparrow^{\prime} \mid \uparrow^{\prime}\right\rangle .
$$

Now since,

$$
\left\langle i S|\hat{P}|\left(X^{\prime}+i Y^{\prime}\right)\right\rangle=i\left\langle S|\hat{P}| X^{\prime}\right\rangle-\left\langle S|\hat{P}| Y^{\prime}\right\rangle=\hat{P}\left(i \hat{r}_{1}-\hat{r}_{2}\right),
$$

we can write

$$
\begin{gathered}
-\left\{\frac{a_{k_{+}} b_{k_{-}}}{\sqrt{2}}\left[\left\langle i S|\hat{P}|\left(X^{\prime}+i Y^{\prime}\right)\right\rangle\left\langle\downarrow^{\prime} \mid \downarrow^{\prime}\right\rangle\right]\right\} \\
=-\left[\frac{a_{k_{+}} b_{k_{-}}}{\sqrt{2}} \hat{P}\left(i \hat{r}_{1}-\hat{r}_{2}\right)\left\langle\downarrow^{\prime} \mid \downarrow^{\prime}\right\rangle\right] .
\end{gathered}
$$

Similarly, we get

$$
\left|Z^{\prime}\right\rangle=\sin \theta \cos \phi|X\rangle+\sin \theta \sin \phi|Y\rangle+\cos \theta|Z\rangle,
$$

so that

$$
\begin{aligned}
\left\langle Z^{\prime}|\hat{P}| i S\right\rangle & =i\left\langle Z^{\prime}|\hat{P}| S\right\rangle \\
& =i \hat{P}\{\sin \theta \cos \phi \hat{i}+\sin \theta \sin \phi \hat{j}+\cos \theta \hat{k}\} \\
& =i \hat{P} \hat{r}_{3},
\end{aligned}
$$

where

$$
\hat{r}_{3}=\hat{i} \sin \theta \cos \phi+\hat{j} \sin \theta \sin \phi+\hat{k} \cos \theta .
$$

Thus,

$$
c_{k_{+}} a_{k_{-}}\left\langle Z^{\prime}|\hat{P}| i S\right\rangle\left\langle\downarrow^{\prime} \mid \uparrow^{\prime}\right\rangle=c_{k_{+}} a_{k_{-}} i \hat{P} \hat{r}_{3}\left\langle\downarrow^{\prime} \mid \uparrow^{\prime}\right\rangle .
$$

Similarly, we can write

$$
c_{k_{-}} a_{k_{+}}\left\langle i S|\hat{P}| Z^{\prime}\right\rangle\left\langle\downarrow^{\prime} \mid \uparrow^{\prime}\right\rangle=c_{k_{-}} a_{k_{+}} i \hat{P} \hat{r}_{3}\left\langle\downarrow^{\prime} \mid \uparrow^{\prime}\right\rangle .
$$

Therefore, we obtain

$$
\begin{aligned}
& \frac{a_{k_{-}} b_{k_{+}}}{\sqrt{2}}\left[\left\langle\left(X^{\prime}-i Y^{\prime}\right)|\hat{P}| S\right\rangle\left\langle\uparrow^{\prime} \mid \uparrow^{\prime}\right\rangle\right]-\frac{a_{k_{+}} b_{k_{-}}}{\sqrt{2}}\left[\left\langle i S|\hat{P}|\left(X^{\prime}+i Y^{\prime}\right)\right\rangle\right. \\
& \left.\quad \times\left\langle\downarrow^{\prime} \mid \downarrow^{\prime}\right\rangle\right] \\
& =\frac{\hat{P}}{\sqrt{2}}\left(-a_{k_{+}} b_{k_{-}}\left\langle\downarrow^{\prime} \mid \downarrow^{\prime}\right\rangle+a_{k_{-}} b_{k_{+}}\left\langle\uparrow \mid \uparrow^{\prime}\right\rangle\right)\left(i \hat{r}_{1}-\hat{r}_{2}\right) .
\end{aligned}
$$

Also, we can write

$$
\begin{gathered}
c_{k_{+}} a_{k_{-}}\left\langle Z^{\prime}|\hat{P}| i S\right\rangle\left\langle\downarrow^{\prime} \mid \uparrow^{\prime}\right\rangle+c_{k_{-}} a_{k_{+}}\left\langle i S|\hat{P}| Z^{\prime}\right\rangle\left\langle\downarrow^{\prime} \mid \uparrow^{\prime}\right\rangle \\
=i \hat{P}\left(c_{k_{+}} a_{k_{-}}+c_{k_{-}} a_{k_{+}}\right) \hat{r}_{3}\left[\left\langle\downarrow^{\prime} \mid \downarrow^{\prime}\right\rangle\right] .
\end{gathered}
$$

By combining Eqs. (23) and (24), we find

$$
\begin{aligned}
\hat{p}_{C V}(\vec{k})= & \frac{\hat{P}}{\sqrt{2}}\left(i \hat{r}_{1}-\hat{r}_{2}\right)\left[\left(b_{k_{+}} a_{k_{-}}\right)\left\langle\uparrow^{\prime} \mid \uparrow^{\prime}\right\rangle-\left(b_{k_{-}} a_{k_{+}}\right)\left\langle\downarrow^{\prime} \mid \downarrow^{\prime}\right\rangle\right] \\
& +i \hat{P} \hat{r}_{3}\left(c_{k_{+}} a_{k_{-}}-c_{k_{-}} a_{k_{+}}\right)\left\langle\downarrow^{\prime} \mid \uparrow^{\prime}\right\rangle .
\end{aligned}
$$

From the above relations, we obtain

$$
\begin{aligned}
& \uparrow^{\prime}=e^{-i \phi / 2} \cos (\theta / 2) \uparrow+e^{i \phi / 2} \sin (\theta / 2) \downarrow, \\
& \downarrow^{\prime}=-e^{-i \phi / 2} \sin (\theta / 2) \uparrow+e^{i \phi / 2} \cos (\theta / 2) \downarrow .
\end{aligned}
$$

Therefore,

$$
\begin{aligned}
\left\langle\downarrow^{\prime} \mid \uparrow^{\prime}\right\rangle_{x}= & -\sin (\theta / 2) \cos (\theta / 2)\langle\uparrow \mid \uparrow\rangle_{x}+e^{-i \phi} \cos ^{2}(\theta / 2)\langle\downarrow \mid \uparrow\rangle_{x} \\
& -e^{i \phi} \sin ^{2}(\theta / 2)\langle\uparrow \mid \downarrow\rangle_{x}+\sin (\theta / 2) \cos (\theta / 2)\langle\downarrow \mid \downarrow\rangle_{x}
\end{aligned}
$$

However, we know from above that

$$
\langle\uparrow \mid \uparrow\rangle_{x}=0, \quad\langle\downarrow \mid \uparrow\rangle=\frac{1}{2}, \quad\langle\downarrow \mid \uparrow\rangle_{x}=\frac{1}{2} \quad \text { and }\langle\downarrow \mid \downarrow\rangle_{x}=0 .
$$

Thus, from Eq. (27), we get

$$
\begin{aligned}
\left\langle\downarrow^{\prime} \mid \uparrow^{\prime}\right\rangle_{x}= & \frac{1}{2}\left[e^{-i \phi} \cos ^{2}(\theta / 2)-e^{i \phi} \sin ^{2}(\theta / 2)\right] \\
= & \frac{1}{2}\left[(\cos \phi-i \sin \phi) \cos ^{2}(\theta / 2)-(\cos \phi\right. \\
& \left.+i \sin \phi) \sin ^{2}(\theta / 2)\right]=\frac{1}{2}[\cos \phi \cos \theta-i \sin \phi] .
\end{aligned}
$$

Similarly, we obtain

$$
\begin{aligned}
\left\langle\downarrow^{\prime} \mid \uparrow^{\prime}\right\rangle_{y}= & \frac{1}{2}[i \cos \phi+\sin \phi \cos \theta] \\
& \text { and }\left\langle\downarrow^{\prime} \mid \uparrow^{\prime}\right\rangle_{z}=\frac{1}{2}[-\sin \theta] .
\end{aligned}
$$

Therefore,

$$
\begin{aligned}
\left\langle\downarrow^{\prime} \mid \uparrow^{\prime}\right\rangle= & \hat{i}\left\langle\downarrow^{\prime} \mid \uparrow^{\prime}\right\rangle_{x}+\hat{j}\left\langle\downarrow^{\prime} \mid \uparrow^{\prime}\right\rangle_{y}+\hat{k}\left\langle\downarrow^{\prime} \mid \uparrow^{\prime}\right\rangle_{z} \\
= & \frac{1}{2}[(\cos \theta \cos \phi-i \sin \phi) \hat{i}+(i \cos \phi \\
& +\sin \phi \cos \theta) \hat{j}-\sin \theta \hat{k}]=\frac{1}{2}\{[(\cos \theta \cos \phi) \hat{i} \\
& +(\sin \phi \cos \theta) \hat{j}-\sin \theta \hat{k}]+i[-\hat{i} \sin \phi \\
& +\hat{j} \cos \phi]\}=\frac{1}{2}\left[\hat{r}_{1}+i \hat{r}_{2}\right]=-\frac{1}{2} i\left[i \hat{r}_{1}-\hat{r}_{2}\right] .
\end{aligned}
$$

Similarly, we can write

$$
\begin{aligned}
\left\langle\uparrow^{\prime} \mid \uparrow^{\prime}\right\rangle= & \frac{1}{2}[\hat{i} \sin \theta \cos \phi+\hat{j} \sin \theta \sin \phi+\hat{k} \cos \theta]=\frac{1}{2} \hat{r}_{3} \\
& \text { and }\left\langle\downarrow^{\prime} \mid \downarrow^{\prime}\right\rangle=-\frac{1}{2} \hat{r}_{3} .
\end{aligned}
$$

By using the above results and following Eq. (25), we can write 


$$
\begin{aligned}
\hat{p}_{C V}(\vec{k})= & \frac{\hat{P}}{\sqrt{2}}\left(i \hat{r}_{1}-\hat{r}_{2}\right)\left[\left(a_{k_{-}} b_{k_{+}}\right)\left\langle\uparrow^{\prime} \mid \uparrow^{\prime}\right\rangle-\left(b_{k_{-}} a_{k_{+}}\right)\left\langle\downarrow^{\prime} \mid \downarrow^{\prime}\right\rangle\right] \\
& +i \hat{P} \hat{r}_{3}\left[\left(c_{k_{+}} a_{k_{-}}-c_{k_{-}} a_{k_{+}}\right)\left\langle\downarrow^{\prime} \mid \uparrow^{\prime}\right\rangle\right] \\
= & \frac{\hat{P}}{2} \hat{r}_{3}\left(i \hat{r}_{1}-\hat{r}_{2}\right)\left[\left(\frac{a_{k_{-}} b_{k_{+}}}{\sqrt{2}}+\frac{b_{k_{-}} a_{k_{+}}}{\sqrt{2}}\right)\right] \\
& +\frac{\hat{P}}{2} \hat{r}_{3}\left(i \hat{r}_{1}-\hat{r}_{2}\right)\left[\left(c_{k_{+}} a_{k_{-}}+c_{k_{-}} a_{k_{+}}\right)\right] .
\end{aligned}
$$

Thus,

$\hat{p}_{C V}(\vec{k})=\frac{\hat{P}}{2} \hat{r}_{3}\left(i \hat{r}_{1}-\hat{r}_{2}\right)\left[a_{k_{+}}\left(\frac{b_{k_{-}}}{\sqrt{2}}+c_{k_{-}}\right)+a_{k_{-}}\left(\frac{b_{k_{+}}}{\sqrt{2}}+c_{k_{+}}\right)\right]$.

We can write that

$$
\begin{aligned}
\left|\hat{r}_{1}\right|= & \left|\hat{r}_{2}\right|=\left|\hat{r}_{3}\right|=1 \\
& \quad \text { also } \hat{P}_{3}=\hat{P}_{x} \sin \theta \cos \phi \hat{i}+\hat{P}_{y} \sin \theta \sin \phi \hat{j} \\
& +\hat{P}_{z} \cos \theta \hat{k},
\end{aligned}
$$

where

$$
\begin{aligned}
& \hat{P}=\langle S|\hat{P}| X\rangle=\langle S|\hat{P}| Y\rangle=\langle S|\hat{P}| Z\rangle \\
& \langle S|\hat{P}| X\rangle=\int u_{C}^{*}(0, \vec{r}) \hat{P} u_{V X}(0, \vec{r}) d^{3} r=\hat{P}_{C V X}(0) \\
& \langle S|\hat{P}| Y\rangle=\hat{P}_{C V Y}(0) \quad \text { and }\langle S|\hat{P}| Z\rangle=\hat{P}_{C V Z}(0)
\end{aligned}
$$

Thus,

$$
\hat{P}=\hat{P}_{C V X}(0)=\hat{P}_{C V Y}(0)=\hat{P}_{C V Z}(0)=\hat{P}_{C V}(0),
$$

where

$$
\hat{P}_{C V}(0) \equiv \int u_{c}^{*}(0, \vec{r}) \hat{P} u_{V}(0, \vec{r}) d^{3} r \equiv \hat{P}
$$

For a plane polarized light wave, we have the polarization vertor $\vec{\varepsilon}_{s}=\hat{k}$, when the light wave vertor is traveling along the $z$ axis. Therefore, for a plane polarized light wave, we have considered $\vec{\varepsilon}_{s}=\hat{k}$.

Then, from Eq. (29),

$$
\left[\vec{\varepsilon} \cdot \hat{p}_{C V}(\vec{k})\right]=\vec{k} \cdot \frac{\hat{P}}{2} \hat{r}_{3}\left(i \hat{r}_{1}-\hat{r}_{2}\right)[A(\vec{k})+B(\vec{k})] \cos \omega t
$$

and

$$
\begin{aligned}
& A(\vec{k})=a_{k_{-}}\left(\frac{b_{k_{+}}}{\sqrt{2}}+c_{k_{+}}\right), \\
& B(\vec{k})=a_{k+}\left(\frac{b_{k_{-}}}{\sqrt{2}+c_{k-}}\right) .
\end{aligned}
$$

Thus,

$$
\begin{aligned}
\left|\vec{\varepsilon} \cdot \hat{p}_{c v}(\vec{k})\right|^{2} & =\left|\hat{k} \cdot \frac{\hat{P}}{2} \hat{r}_{3}\right|{ }^{2}\left|i \hat{r}_{1}-\hat{r}_{2}\right|^{2}[A(\vec{k})+B(\vec{k})]^{2} \cos ^{2} \omega t \\
& =\frac{1}{4}\left|\hat{P}_{z} \cos \theta\right|^{2}[A(\vec{k})+B(\vec{k})]^{2} \cos ^{2} \omega t
\end{aligned}
$$

So, the average value of $\left|\vec{\varepsilon} \cdot \hat{p}_{c v}(\vec{k})\right|^{2}$ for a plane polarized light wave is given by

$$
\begin{aligned}
\langle| \vec{\varepsilon} & \left.\left.\cdot \hat{p}_{c v}(\vec{k})\right|^{2}\right\rangle_{\mathrm{av}} \\
& =\frac{2}{4}\left|\hat{P}_{z}\right|^{2}[A(\vec{k})+B(\vec{k})]^{2}\left(\int_{0}^{2 \pi} d \phi \int_{0}^{\pi} \cos ^{2} \theta \sin \theta d \theta\right)\left(\frac{1}{2}\right) \\
& =\frac{2 \pi}{3}\left|\hat{P}_{z}\right|^{2}[A(\vec{k})+B(\vec{k})]^{2}
\end{aligned}
$$

where

$$
\left|\hat{P}_{z}\right|^{2}=\left(\frac{1}{2}\right)\left|\vec{k} \cdot \hat{p}_{c v}(0)\right|^{2}
$$

and

$$
\left|\vec{k} \cdot \hat{p}_{c v}(0)\right|^{2}=\frac{m^{2}}{4 m_{r}} \frac{E_{g_{0}}\left(E_{g_{0}}+\Delta\right)}{\left(E_{g_{0}}+\frac{2}{3} \Delta\right)} .
$$

We shall express $A(\vec{k})$ and $B(\vec{k})$ in terms of constants of the energy spectra in the following way: By substituting $a_{k_{ \pm}}$, $b_{k_{ \pm}}, c_{k_{ \pm}}$, and $\gamma_{0 k_{ \pm}}$in $A(\vec{k})$ and $B(\vec{k})$ in Eq. (31), we get

$$
\begin{aligned}
A(\vec{k})= & \beta\left(t+\frac{\rho}{\sqrt{2}}\right)\left[\left(\frac{E_{g_{0}}}{E_{g_{0}}+\delta^{\prime}}\right) \gamma_{0 k_{+}}^{2}\right. \\
& \left.-\gamma_{0 k_{+}}^{2} \gamma_{0 k_{-}}^{2}\left(\frac{E_{g_{0}}-\delta^{\prime}}{E_{g_{0}}+\delta^{\prime}}\right)\right]^{1 / 2}, \\
B(\vec{k})= & \beta\left(t+\frac{\rho}{\sqrt{2}}\right)\left[\left(\frac{E_{g_{0}}}{E_{g_{0}}+\delta^{\prime}}\right) \gamma_{0 k_{-}}^{2}\right. \\
& \left.-\gamma_{0 k_{+}}^{2} \gamma_{0 k_{-}}^{2}\left(\frac{E_{g_{0}}-\delta^{\prime}}{E_{g_{0}}+\delta^{\prime}}\right)\right]^{1 / 2},
\end{aligned}
$$

in which

$$
\begin{aligned}
\gamma_{0 k_{+}}^{2} & \equiv \frac{\xi_{1 k}-E_{g_{0}}}{2\left(\xi_{1 k}+\delta^{\prime}\right)} \equiv \frac{1}{2}\left[1-\left(\frac{E_{g_{0}}+\delta^{\prime}}{\xi_{1 k}+\delta^{\prime}}\right)\right] \text { and } \gamma_{0 k_{-}}^{2} \\
& \equiv \frac{\xi_{1 k}+E_{g_{0}}}{2\left(\xi_{1 k}+\delta^{\prime}\right)} \equiv \frac{1}{2}\left[1+\left(\frac{E_{g_{0}}-\delta^{\prime}}{\xi_{1 k}+\delta^{\prime}}\right)\right]
\end{aligned}
$$

By substituting $x \equiv \xi_{1 k}+\delta^{\prime}$ in $\gamma_{0 k_{ \pm}}^{2}$, we can write 


$$
\begin{aligned}
A(\vec{k})= & \beta\left(t+\frac{\rho}{\sqrt{2}}\right)\left[\left(\frac{E_{g_{0}}}{E_{g_{0}}+\delta^{\prime}}\right) \frac{1}{2}\left(1-\frac{E_{g_{0}}+\delta^{\prime}}{x}\right)\right. \\
& -\frac{1}{4}\left(\frac{E_{g_{0}}-\delta^{\prime}}{E_{g_{0}}+\delta^{\prime}}\right)\left(1-\frac{E_{g_{0}}+\delta^{\prime}}{x}\right) \\
& \left.\times\left(1+\frac{E_{g_{0}}-\delta^{\prime}}{x}\right)\right]^{1 / 2} .
\end{aligned}
$$

Thus,

$$
A(\vec{k})=\frac{\beta}{2}\left(t+\frac{\rho}{\sqrt{2}}\right)\left\{1-\frac{2 a_{0}}{x}+\frac{a_{1}}{x^{2}}\right\}^{1 / 2},
$$

where

$$
a_{0} \equiv\left(E_{g_{0}}^{2}+\delta^{\prime 2}\right)\left(E_{g_{0}}+\delta^{\prime}\right)^{-1} \quad \text { and } a_{1} \equiv\left(E_{g_{0}}-\delta^{\prime}\right)^{2} .
$$

After tedious algebra, one can show that

$$
\begin{aligned}
A(\vec{k})= & \frac{\beta}{2}\left(t+\frac{\rho}{\sqrt{2}}\right)\left(E_{g_{0}}-\delta^{\prime}\right)\left[\frac{1}{\xi_{1 k}+\delta^{\prime}}\right. \\
& \left.-\frac{1}{E_{g_{0}}+\delta^{\prime}}\right]^{1 / 2}\left[\frac{1}{\xi_{1 k}+\delta^{\prime}}-\frac{\left(E_{g_{0}}+\delta^{\prime}\right)}{\left(E_{g_{0}}-\delta^{\prime}\right)^{2}}\right]^{1 / 2} .
\end{aligned}
$$

Similarly, from Eq. (36), we can write

$$
\begin{aligned}
B(\vec{k})= & \beta\left(t+\frac{\rho}{\sqrt{2}}\right)\left[\left(\frac{E_{g_{0}}}{E_{g_{0}}+\delta^{\prime}}\right) \frac{1}{2}\left(1+\frac{E_{g_{0}}-\delta^{\prime}}{x}\right)\right. \\
- & \frac{1}{4}\left(\frac{E_{g_{0}}-\delta^{\prime}}{E_{g_{0}}+\delta^{\prime}}\right)\left(1-\frac{E_{g_{0}}+\delta^{\prime}}{x}\right) \\
& \left.\times\left(1+\frac{E_{g_{0}}-\delta^{\prime}}{x}\right)\right]^{1 / 2} .
\end{aligned}
$$

So that finally, we get

$$
B(\vec{k})=\frac{\beta}{2}\left(t+\frac{\rho}{\sqrt{2}}\right)\left(1+\frac{E_{g_{0}}-\delta^{\prime}}{\xi_{1 k}+\delta^{\prime}}\right) .
$$

By using Eqs. (33), (34), (37), and (38), we can write

$$
\begin{aligned}
\left(\frac{|e| A_{0}}{2 m}\right)^{2} & \frac{\left\langle\left|\vec{\varepsilon} \cdot \hat{p}_{c v}(\vec{k})\right|^{2}\right\rangle_{\mathrm{av}}}{E_{c}(\vec{k})-E_{v}(\vec{k})} \\
= & \left(\frac{|e| A_{0}}{2 m}\right)^{2} \frac{2 \pi}{3}\left|\vec{k} \cdot \hat{p}_{c v}(0)\right|^{2} \frac{\beta^{2}}{4}\left(t+\frac{\rho}{\sqrt{2}}\right)^{2} \frac{1}{\xi_{1 k}} \\
& \times\left\{\left(1+\frac{E_{g_{0}}-\delta^{\prime}}{\xi_{1 k}+\delta^{\prime}}\right)+\left(E_{g_{0}}-\delta^{\prime}\right)\right. \\
& \times\left[\frac{1}{\xi_{1 k}+\delta^{\prime}}-\frac{1}{E_{g_{0}}+\delta^{\prime}}\right]^{1 / 2} \\
& \left.\times\left[\frac{1}{\xi_{1 k}+\delta^{\prime}}-\frac{E_{g_{0}}+\delta^{\prime}}{\left(E_{g_{0}}-\delta^{\prime}\right)^{2}}\right]^{1 / 2}\right\}^{2} .
\end{aligned}
$$

By following the work of Nag, ${ }^{77}$ it can be shown that

$$
A_{0}^{2}=\frac{I \lambda^{2}}{2 \pi^{2} c^{3} \sqrt{\varepsilon_{s c} \varepsilon_{0}}},
$$

where $I$ is the light intensity of wavelength $\lambda$ and $c$ is the velocity of light. Thus, the simplified electron energy spectrum in III-V, ternary, and quaternary materials up to the second order in the presence of light waves can approximately be written as

$$
\frac{\hbar^{2} k^{2}}{2 m_{c}}=\beta_{0}(E, \lambda),
$$

where

$$
\beta_{0}(E, \lambda) \equiv\left[\gamma(E)-\theta_{0}(E, \lambda)\right],
$$

$\theta_{0}(E, \lambda)$

$$
\begin{aligned}
\equiv & \frac{|e|^{2}}{96 m_{r} \pi c^{3}} \frac{I \lambda^{2}}{\sqrt{\varepsilon_{s c} \varepsilon_{0}}} \frac{E_{g_{0}}\left(E_{g_{0}}+\Delta\right)}{\left(E_{g_{0}}+\frac{2}{3} \Delta\right)} \frac{\beta^{2}}{4} \\
& \times\left(t+\frac{\rho}{\sqrt{2}}\right)^{2} \frac{1}{\phi_{0}(E)}\left\{\left(1+\frac{E_{g_{0}}-\delta^{\prime}}{\phi_{0}(E)+\delta^{\prime}}\right)+\left(E_{g_{0}}-\delta^{\prime}\right)\right. \\
& \times\left[\frac{1}{\phi_{0}(E)+\delta^{\prime}}-\frac{1}{E_{g_{0}}+\delta^{\prime}}\right]^{1 / 2} \\
& \left.\times\left[\frac{1}{\phi_{0}(E)+\delta^{\prime}}-\frac{E_{g_{0}}+\delta^{\prime}}{\left(E_{g_{0}}-\delta^{\prime}\right)^{2}}\right]^{1 / 2}\right\}^{2},
\end{aligned}
$$

and

$$
\phi_{0}(E) \equiv E_{g_{0}}\left[1+2\left(1+\frac{m^{*}}{m_{v}}\right) \frac{\gamma(E)}{E_{g_{0}}}\right]^{1 / 2} .
$$

Thus, under the limiting condition of $\vec{k} \rightarrow 0$, from Eq. (41), we observe that $E \neq 0$ and is positive. Therefore, in the presence of external light waves, the energy of the electron does not tend to zero when $\vec{k} \rightarrow 0$, whereas for the unperturbed three band model of Kane, $I(E)=\left[\hbar^{2} k^{2} /\left(2 m^{*}\right)\right]$, in which $E$ $\rightarrow 0$ for $\vec{k} \rightarrow 0$. As the conduction band is taken as the reference level of energy, therefore, the lowest positive value of $E$ for $\vec{k} \rightarrow 0$ provides the increased band gap $\left(\Delta E_{g}\right)$ of the semiconductor due to photon excitation. The values of the increased band gap can be obtained by computer iteration processes for various values of $I$ and $\lambda$.

\section{Special Cases}

(1) For the two band model of Kane, we have $\Delta \rightarrow 0$. Under this condition, $\gamma(E) \rightarrow E(1+a E)=\hbar^{2} k^{2} / 2 m^{*}$. Since $\beta$ $\rightarrow 1, t \rightarrow 1, \rho \rightarrow 0$, and $\delta^{\prime} \rightarrow 0$ for $\Delta \rightarrow 0$, from Eq. (41), we can write the energy spectrum of III-V, ternary, and quaternary materials in the presence of external photoexcitation whose unperturbed conduction electrons obey the two band model of Kane as

$\frac{\hbar^{2} k^{2}}{2 m_{c}}=\omega_{0}(E, \lambda)$,

where 


$$
\begin{aligned}
& \omega_{0}(E, \lambda) \equiv E(1+a E)-B_{0}(E, \lambda), \\
& B_{0}(E, \lambda) \equiv \frac{|e|^{2} I \lambda^{2} E_{g_{0}}}{384 \pi c^{3} m_{r} \sqrt{\varepsilon_{s c} \varepsilon_{0}}} \frac{1}{\phi_{1}(E)}\left\{\left[1+\frac{E_{g_{0}}}{\phi_{1}(E)}\right]\right. \\
&\left.+E_{g_{0}}\left[\frac{1}{\phi_{1}(E)}-\frac{1}{E_{g_{0}}}\right]\right\}^{2}, \\
& \phi_{1}(E) \equiv E_{g_{0}}\left[1+\frac{2 m^{*}}{m_{r}} a E(1+a E)\right]^{1 / 2} .
\end{aligned}
$$

(2) In the case of relatively wide band gap semiconductors, we can write $a \rightarrow 0, b \rightarrow 0, c \rightarrow 0$, and $\gamma(E) \rightarrow E$. Thus, from Eq. (42), we can find

$$
\frac{\hbar^{2} k^{2}}{2 m_{c}}=\rho_{0}(E, \lambda),
$$

$$
\rho_{0}(E, \lambda) \equiv E-\frac{|e|^{2} I \lambda^{2}}{96 \pi c^{3} m_{r} \sqrt{\varepsilon_{s c} \varepsilon_{0}}}\left[1+\left(\frac{2 m^{*}}{m_{r}}\right) a E\right]^{-3 / 2} .
$$

\section{B. Formulation of the Einstein relation in the presence of light waves in QWs of III-V, ternary, and quaternary materials}

The DMR can, in general, ${ }^{5}$ be written as

$$
\frac{D}{\mu}=\frac{1}{e} N\left(\frac{\partial N}{\partial \bar{E}_{F}}\right)^{-1}
$$

where $N$ is the electron concentration and $\bar{E}_{F}$ is the Fermi energy. It appears then that the formulation of the DMR requires an expression of electron statistics, which, in turn, is determined by the density-of-states DOS function.

(i) The 1D electron energy spectrum in QWs of III-V, ternary, and quaternary materials, whose unperturbed band structure is defined by the three band model of Kane, in the presence of light waves can be expressed from Eq. (41) as

$$
\frac{\hbar^{2} k_{x}^{2}}{2 m_{c}}=\beta_{0}(E, \lambda)-\left[\frac{\hbar^{2}}{2 m_{c}}\left(\frac{n_{y} \pi}{d_{y}}\right)^{2}+\frac{\hbar^{2}}{2 m_{c}}\left(\frac{n_{z} \pi}{d_{z}}\right)^{2}\right],
$$

where $n_{y}(=1,2,3, \ldots)$ and $n_{z}(=1,2,3, \ldots)$ are the size quantum numbers along the $y$ and $z$ directions, while $d_{y}$ and $d_{z}$ are the nanothickness along the $y$ and $z$ directions respectively. The subband energies $\left(E_{n}\right)$ can be expressed as

$$
\beta_{0}\left(E_{n}, \lambda\right)=\left[\frac{\hbar^{2}}{2 m_{c}}\left(n_{y} \pi / d_{y}\right)^{2}+\frac{\hbar^{2}}{2 m_{c}}\left(n_{z} \pi / d_{z}\right)^{2}\right] .
$$

The 1D DOS function $\left[N_{1 D}(E, \lambda)\right]$ is given by

$$
\begin{aligned}
N_{1 D}(E, \lambda)= & \left(\frac{\sqrt{2 m_{c}}}{\pi \hbar}\right) \sum_{n_{y}=1}^{n_{y_{\max }}} \sum_{n_{z}=1}^{n_{z_{\max }}} \beta_{0}^{\prime}(E, \lambda)\left[\beta_{0}(E, \lambda)\right. \\
& \left.-\phi\left(n_{y}, n_{z}\right)\right]^{-1 / 2} H\left(E-E_{n}\right),
\end{aligned}
$$

where the prime indicates the differentiation of the differentiable functions with respect to $E$,

$$
\phi\left(n_{y}, n_{z}\right)=\frac{\hbar^{2} \pi^{2}}{2 m_{c}}\left[\left(\frac{n_{y}}{d_{y}}\right)^{2}+\left(\frac{n_{z}}{d_{z}}\right)^{2}\right],
$$

and $H$ is the Heaviside step function. By combining Eq. (48) with the Fermi Dirac occupation probability factor, the 1D electron concentrations $\left(n_{1 D}\right)$ can, thus, be written as

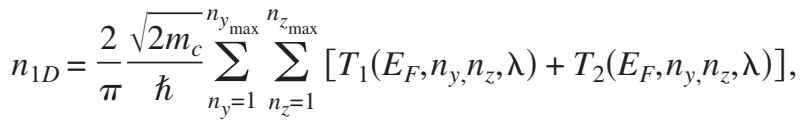

where $T_{1}\left(E_{F}, n_{y}, n_{z}, \lambda\right) \equiv\left[\beta_{0}\left(E_{F}, \lambda\right)-\phi\left(n_{y}, n_{z}\right)\right]^{1 / 2}, E_{F}$ is the Fermi energy in the present case, $T_{2}\left(E_{F}, n_{y}, n_{z}, \lambda\right)$ $\equiv \sum_{r=1}^{s_{0}} Z_{r} T_{1}\left(E_{F}, n_{y}, n_{z}, \lambda\right), \quad Z_{r} \equiv 2\left(k_{B} T\right)^{2 r}(1$ $\left.-2^{1-2 r}\right) \zeta(2 r) \partial^{2 r} / \partial E_{F}^{2 r}, \zeta(2 r)$ is the zeta function of order $2 r$, and $r$ is the set of positive integers whose upper limit is $S_{0}{ }^{78}$ The use of Eqs. (49) and (45) leads to the expression of the 1D DMR in this case as

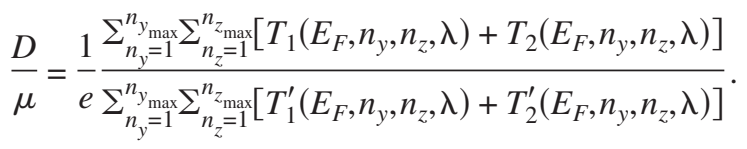

(ii) By using Eq. (42), the expressions for the 1D dispersion relation, the subband energies, the DOS function, and the electron concentration for QWs of III-V, ternary, and quaternary materials, whose unperturbed band structure is defined by the two band model of Kane, can, respectively, be written in the presence of photoexcitation as

$$
\begin{aligned}
& \frac{\hbar^{2} k_{x}^{2}}{2 m_{c}}=\left[\omega_{0}(E, \lambda)-\phi\left(n_{y}, n_{z}\right)\right], \\
& \omega_{0}\left(E_{n}, \lambda\right)=\phi\left(n_{y}, n_{z}\right), \\
& N_{1 D}(E, \lambda)=\left(\frac{\sqrt{2 m_{c}}}{\pi \hbar}\right)_{n_{y}=1}^{n_{y_{\max }}} \sum_{n_{z}=1}^{n_{z_{\max }}} \omega_{0}^{\prime}(E, \lambda)\left[\omega_{0}(E, \lambda)\right. \\
& \text { - } \left.\phi\left(n_{y}, n_{z}\right)\right]^{-1 / 2} H\left(E-E_{n}\right),
\end{aligned}
$$

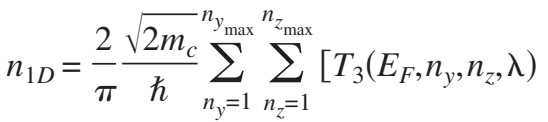

$$
\begin{aligned}
& \left.+T_{4}\left(E_{F}, n_{y}, n_{z}, \lambda\right)\right],
\end{aligned}
$$

where

$$
T_{3}\left(E_{F}, n_{y}, n_{z}, \lambda\right) \equiv\left[\omega_{0}\left(E_{F}, \lambda\right)-\phi\left(n_{y}, n_{z}\right)\right]^{1 / 2},
$$




$$
T_{4}\left(E_{F}, n_{y}, n_{z}, \lambda\right) \equiv \sum_{r=1}^{s_{0}} Z_{r} T_{3}\left(E_{F}, n_{y}, n_{z}, \lambda\right) .
$$

The use of Eqs. (54) and (45) leads to the expression of the DMR in this case as

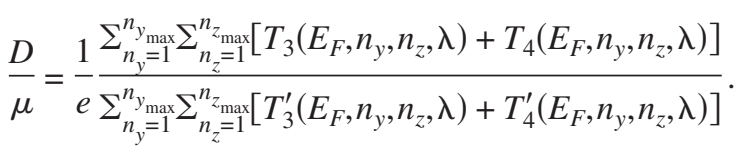

(iii) By using Eq. (43), the expressions for the 1D dispersion relation, the subband energies, the DOS function, and the electron concentration for QWs of III-V, ternary, and quaternary materials, whose unperturbed band structure is defined by the parabolic energy bands, can, respectively, be written in the presence of photoexcitation as

$$
\begin{aligned}
& \frac{\hbar^{2} k_{x}^{2}}{2 m_{c}}=\left[\rho_{0}(E, \lambda)-\phi\left(n_{y}, n_{z}\right)\right], \\
& \rho_{0}\left(E_{n}, \lambda\right)=\phi\left(n_{y}, n_{z}\right),
\end{aligned}
$$

$$
\begin{aligned}
N_{1 D}(E, \lambda)= & \left(\frac{\sqrt{2 m_{c}}}{\pi \hbar}\right) \sum_{n_{y}=1}^{n_{y_{\max }}} \sum_{n_{z}=1}^{n_{z_{\max }}} \rho_{0}^{\prime}(E, \lambda)\left[\rho_{0}(E, \lambda)\right. \\
& \left.-\phi\left(n_{y}, n_{z}\right)\right]^{-1 / 2} H\left(E-E_{n}\right), \\
n_{1 D}= & \frac{2 \sqrt{2 m_{c}}}{\pi \hbar} \sum_{n_{y}=1}^{n_{y_{\max }}} \sum_{n_{z}=1}^{n_{\text {max }}}\left[T_{5}\left(E_{F}, n_{y}, n_{z}, \lambda\right)+T_{6}\left(E_{F}, n_{y}, n_{z}, \lambda\right)\right],
\end{aligned}
$$

where

$$
\begin{aligned}
& T_{5}\left(E_{F}, n_{y}, n_{z}, \lambda\right) \equiv\left[\rho_{0}\left(E_{F}, \lambda\right)-\phi\left(n_{y}, n_{z}\right)\right]^{1 / 2}, \\
& T_{6}\left(E_{F}, n_{y}, n_{z}, \lambda\right) \equiv \sum_{r=1}^{s_{0}} Z_{r} T_{5}\left(E_{F}, n_{y}, n_{z}, \lambda\right) .
\end{aligned}
$$

The use of Eqs. (54) and (45) leads to the expression of the DMR in this case as

$$
\frac{D}{\mu}=\frac{1}{e} \frac{\sum_{n_{y}=1}^{n_{y_{\max }}} \sum_{n_{z}=1}^{n_{z_{\max }}}\left[T_{5}\left(E_{F}, n_{y}, n_{z}, \lambda\right)+T_{6}\left(E_{F}, n_{y}, n_{z}, \lambda\right)\right]}{\sum_{n_{y}=1}^{n_{y_{\max }} \sum \sum_{n_{z}=1}^{n_{z_{m}}}[}\left[T_{5}^{\prime}\left(E_{F}, n_{y}, n_{z}, \lambda\right)+T_{6}^{\prime}\left(E_{F}, n_{y}, n_{z}, \lambda\right)\right]} .
$$

\section{Formulation of the Einstein relation in the absence of light waves in QWs of III-V, ternary, and quaternary materials}

(i) The expressions for the 1D dispersion relation, the subband energies, the DOS function, and the electron concentration for QWs of III-V, ternary, and quaternary materials, whose unperturbed band structure is defined by the three band model of Kane, can, respectively, be written in the absence of photoexcitation as

$$
\begin{aligned}
& \frac{\hbar^{2} k_{x}^{2}}{2 m_{c}}=\left[\gamma(E)-\phi\left(n_{y}, n_{z}\right)\right], \\
& \gamma\left(E_{n}\right)=\phi\left(n_{y}, n_{z}\right), \\
& N_{1 D}(E)=\left(\frac{\sqrt{2 m_{c}}}{\pi \hbar}\right) \sum_{n_{y}=1}^{n_{y_{\max }}} \sum_{n_{z}=1}^{n_{z_{\max }}} \gamma^{\prime}(E)[\gamma(E) \\
& \text { - } \left.\phi\left(n_{y}, n_{z}\right)\right]^{-1 / 2} H\left(E-E_{n}\right) \text {, } \\
& n_{1 D}=\frac{2 \sqrt{2 m_{c}}}{\pi \hbar} \sum_{n_{y}=1}^{n_{y_{\max }}} \sum_{n_{z}=1}^{n_{z_{\max }}}\left[T_{7}\left(E_{F_{0}}, n_{y}, n_{z}\right)+T_{8}\left(E_{F_{0}}, n_{y}, n_{z}\right)\right],
\end{aligned}
$$

where $E_{F_{0}}$ is the Fermi energy in the presence of size quantization as measured from the edge of the conduction band in the vertically upward direction in the absence of external photoexcitation, $T_{7}\left(E_{F_{0}}, n_{y}, n_{z}\right) \equiv\left[\gamma\left(E_{F_{0}}\right)-\phi\left(n_{y}, n_{z}\right)\right]^{1 / 2}$, and $T_{8}\left(E_{F_{0}}, n_{y}, n_{z}\right) \equiv \sum_{r=1}^{s_{0}} Z_{r} T_{7}\left(E_{F_{0}}, n_{y}, n_{z}\right)$. The use of Eqs. (64) and (45) leads to the expression of the DMR in this case as

$$
\frac{D}{\mu}=\frac{1}{e} \frac{\sum_{n_{y}=1}^{n_{y_{\max }}} \sum_{n_{z}=1}^{n_{z_{\max }}}\left[T_{7}\left(E_{F_{0}}, n_{y}, n_{z}\right)+T_{8}\left(E_{F_{0}}, n_{y}, n_{z}\right)\right]}{\sum_{n_{y}=1}^{n_{y} \max } \sum_{n_{z}=1}^{n_{z_{\max }}}\left[T_{7}^{\prime}\left(E_{F_{0}}, n_{y}, n_{z}\right)+T_{8}^{\prime}\left(E_{F_{0}}, n_{y}, n_{z}\right)\right]} .
$$

(ii) The expressions for the 1D dispersion relation, the subband energies, the DOS function, and the electron concentration for QWs of III-V, ternary, and quaternary materials, whose unperturbed band structure is defined by the two band model of Kane, can, respectively, be written in the absence of photoexcitation as

$$
\begin{aligned}
& E(1+a E) \equiv \phi\left(n_{y}, n_{z}\right)+\frac{\hbar^{2} k_{x}^{2}}{2 m_{c}} \\
& E_{n}\left(1+a E_{n}\right) \equiv \phi\left(n_{y}, n_{z}\right), \\
& N_{1 D}(E)=\frac{\sqrt{2 m_{c}}}{\pi \hbar} \sum_{n_{y}=1}^{n_{y_{\max }}} \sum_{n_{z}=1}^{n_{z_{\max }}}[1+2 a E][E(1+a E) \\
& \left.-\phi\left(n_{y}, n_{z}\right)\right]^{1 / 2} H\left(E-E_{n}\right),
\end{aligned}
$$

$$
\begin{aligned}
n_{1 D}= & \frac{2 \sqrt{2 m_{c} \pi k_{B} T}}{h} \sum_{n_{y}=1}^{n_{y_{\max }}} \sum_{n_{z}=1}^{n_{z_{\max }}} \frac{1}{\sqrt{a_{0}}}\left[\left(1+\frac{3}{2} a b_{0}\right) F_{-1 / 2}\left(\eta_{n}\right)\right. \\
& \left.+\frac{3}{4} a k_{B} T F_{1 / 2}\left(\eta_{n}\right)\right]
\end{aligned}
$$

where $a_{0}=\left[1+a \phi\left(n_{y}, n_{z}\right)\right], \quad b_{0}=\phi\left(n_{y}, n_{z}\right)\left[1+a \phi\left(n_{y}, n_{z}\right)\right]^{-1}$, $\eta_{n} \equiv\left(E_{F_{0}}-b_{0}\right) / k_{B} T$, and $F_{t_{0}}\left(\eta_{n}\right)$ is one-parameter FermiDirac integral of order $t_{0}$ which can be written as ${ }^{79}$

$$
F_{t_{0}}\left(\eta_{n}\right)=\left[\frac{1}{\Gamma\left(t_{0}+1\right)}\right] \int_{0}^{\infty} y^{t_{0}}\left(1+e^{(y-\eta)}\right)^{-1} d y, \quad y>-1,
$$

where $\Gamma\left(t_{0}+1\right)$ is the complete gamma function, or for all $t$, analytically continued as a complex contour integral around the negative axis, 


$$
F_{t_{0}}\left(\eta_{n}\right)=A_{t_{0}} \int_{-\infty}^{(0+)} y^{t_{0}}[1+\exp (-y-\eta)]^{-1} d y
$$

in which $A_{t_{0}}=\Gamma\left(-t_{0}\right) / 2 \pi \sqrt{-1}$. The use of Eqs. (69) and (45) leads to the expression of the DMR in this case as

$$
\frac{D}{\mu}=\frac{k_{B} T}{e} \frac{\sum_{n_{y}=1} \sum_{n_{z}=1}^{n_{y_{\max }} n_{z_{\max }}} \frac{1}{\sqrt{a_{0}}}\left[\left(1+\frac{3}{2} a b_{0}\right) F_{-1 / 2}\left(\eta_{n}\right)+\frac{3}{4} a k_{B} T F_{1 / 2}\left(\eta_{n}\right)\right]}{\sum_{n_{y}=1} \sum_{n_{\max }=1}^{n_{z_{\max }}} \frac{1}{\sqrt{a_{0}}}\left[\left(1+\frac{3}{2} a b_{0}\right) F_{-3 / 2}\left(\eta_{n}\right)+\frac{3}{4} a k_{B} T F_{-1 / 2}\left(\eta_{n}\right)\right]} .
$$

(iii) Under the condition of $a \rightarrow 0$ as parabolic energy bands, the expressions for the 1D dispersion relation, the subband energies, the DOS function, the electron concentration, and the DMR can be written as

$$
\begin{aligned}
& E=\phi\left(n_{y}, n_{z}\right)+\frac{\hbar^{2} k_{x}^{2}}{2 m_{c}}, \\
& E_{n}=\phi\left(n_{y}, n_{z}\right), \\
& n_{1 D}=\frac{2 \sqrt{2 m_{c} \pi k_{B} T}}{h} \sum_{n_{y}=1}^{n_{y_{\max }}} \sum_{n_{z}=1}^{n_{z_{\max }}}\left[F_{-1 / 2}\left(\eta_{n}\right)\right], \\
& \frac{D}{\mu}=\frac{k_{B} T}{e} \frac{\sum_{n_{y}=1}^{n_{y_{\max }}} \sum_{n_{z}=1}^{n_{z_{\max }}}\left[F_{-1 / 2}\left(\eta_{n}\right)\right]}{\sum_{n_{y}=1}^{n_{y_{\max }} \sum_{n_{z}=1}^{n_{z_{\max }}}\left[F_{-3 / 2}\left(\eta_{n}\right)\right]} .}
\end{aligned}
$$$$
N_{1 D}(E)=\frac{\sqrt{2 m_{c}}}{\pi \hbar} \sum_{n_{y}=1}^{n_{y_{\max }}} \sum_{n_{z}=1}^{n_{z_{\max }}}\left[E-\phi\left(n_{y}, n_{z}\right)\right]^{-1 / 2} H\left(E-E_{n}\right),
$$

\section{Formulation of the Einstein relation in the presence of light waves in bulk specimens of III-V, ternary, and quaternary materials}

(i) By using Eq. (41), the DOS function for III-V, ternary, and quaternary materials in the absence of size quantization and in the presence of light waves whose unperturbed conduction electrons obey the three band model of Kane can be expressed as

$D_{0}(E)=4 \pi\left(\frac{2 m_{c}}{h^{2}}\right)^{3 / 2} \sqrt{\beta_{0}(E, \lambda)} \beta_{0}^{\prime}(E, \lambda)$.

By combining Eq. (78) with the Fermi-Dirac occupation probability factor and by using the generalized Sommerfeld's lemma, ${ }^{78}$ the electron concentration $\left(n_{0}\right)$ can be written as

$$
n_{0}=\left(3 \pi^{2}\right)^{-1}\left(\frac{2 m_{c}}{\hbar^{2}}\right)^{3 / 2}\left[M_{1}\left(E_{F B}, \lambda\right)+N_{1}\left(E_{F B}, \lambda\right)\right]
$$

where $\quad M_{1}\left(E_{F B}, \lambda\right) \equiv\left[\beta_{0}\left(E_{F B}, \lambda\right)\right]^{3 / 2}, \quad N_{1}\left(E_{F B}, \lambda\right)$ $\equiv \sum_{r=1}^{s_{0}} Z_{r} M_{1}\left(E_{F B}, \lambda\right)$, and $E_{F B}$ is the Fermi energy as measured from the edge of the conduction band in the vertically upward direction in the absence of size quantization and in the presence of photoexcitation. By using the Eqs. (79) and (45), we get

$$
\frac{D}{\mu}=\frac{1}{e} \frac{\left[M_{1}\left(E_{F B}, \lambda\right)+N_{1}\left(E_{F B}, \lambda\right)\right]}{\left[M_{1}^{\prime}\left(E_{F B}, \lambda\right)+N_{1}^{\prime}\left(E_{F B}, \lambda\right)\right]} .
$$

(ii) The expressions of $n_{0}$ and DMR for III-V, ternary, and quaternary materials in the presence of light waves whose unperturbed conduction electrons obey the two band model of Kane can be expressed as

$$
n_{0}=\left(3 \pi^{2}\right)^{-1}\left(\frac{2 m_{c}}{\hbar^{2}}\right)^{3 / 2}\left[M_{2}\left(E_{F B}, \lambda\right)+N_{2}\left(E_{F B}, \lambda\right)\right]
$$

and

$$
\frac{D}{\mu}=\frac{1}{e} \frac{\left[M_{2}\left(E_{F B}, \lambda\right)+N_{2}\left(E_{F B}, \lambda\right)\right]}{\left[M_{2}^{\prime}\left(E_{F B}, \lambda\right)+N_{2}^{\prime}\left(E_{F B}, \lambda\right)\right]}
$$

where

$$
\begin{aligned}
M_{2}\left(E_{F B}, \lambda\right) & \equiv\left[\omega_{0}\left(E_{F B}, \lambda\right)\right]^{3 / 2}, \quad N_{2}\left(E_{F B}, \lambda\right) \\
& \equiv \sum_{r=1}^{s_{0}} Z_{r} M_{2}\left(E_{F B}, \lambda\right) .
\end{aligned}
$$

(iii) The expressions of $n_{1 D}$ and DMR for III-V, ternary, and quaternary materials in the presence of light waves whose unperturbed conduction electrons obey the parabolic energy bands can be expressed as

$$
n_{0}=\left(3 \pi^{2}\right)^{-1}\left(\frac{2 m_{c}}{\hbar^{2}}\right)^{3 / 2}\left[M_{3}\left(E_{F B}, \lambda\right)+N_{3}\left(E_{F B}, \lambda\right)\right]
$$

and

$$
\frac{D}{\mu}=\frac{1}{e} \frac{\left[M_{3}\left(E_{F B}, \lambda\right)+N_{3}\left(E_{F B}, \lambda\right)\right]}{\left[M_{3}^{\prime}\left(E_{F B}, \lambda\right)+N_{3}^{\prime}\left(E_{F B}, \lambda\right)\right]},
$$

where

$$
\begin{aligned}
& M_{3}\left(E_{F B}, \lambda\right) \equiv\left[\rho_{0}\left(E_{F B}, \lambda\right)\right]^{3 / 2}, \\
& N_{3}\left(E_{F B}, \lambda\right) \equiv \sum_{r=1}^{s_{0}} Z_{r} M_{3}\left(E_{F B}, \lambda\right) .
\end{aligned}
$$




\section{E. Formulation of the Einstein relation in the absence of light waves in bulk specimens of III-V, ternary, and quaternary materials}

(i) In the absence of external photoexcitations, the expressions of the $n_{0}$ and the DMR in accordance with the three band model of Kane assume the forms

$$
\begin{aligned}
& n_{0}=\left(3 \pi^{2}\right)^{-1}\left(\frac{2 m_{c}}{\hbar^{2}}\right)^{3 / 2}\left[M_{4}\left(E_{F B 0}\right)+N_{4}\left(E_{F B 0}\right)\right], \\
& \frac{D}{\mu}=\frac{1}{e} \frac{\left[M_{4}\left(E_{F B 0}\right)+N_{4}\left(E_{F B 0}\right)\right]}{\left[M_{4}^{\prime}\left(E_{F B 0}\right)+N_{4}^{\prime}\left(E_{F B 0}\right)\right]},
\end{aligned}
$$

where $M_{4}\left(E_{F B 0}\right) \equiv\left[\gamma\left(E_{F B 0}\right)\right]^{3 / 2}, E_{F B 0}$ is the Fermi energy as measured from the edge of the conduction band in the vertically upward direction in the absence of any field, and $N_{4}\left(E_{F B 0}\right)=\sum_{r=1}^{s_{0}} Z_{r} M_{4}\left(E_{F B 0}\right)$.

(ii) In accordance with the two band model of Kane, the corresponding expressions of the $n_{0}$ and DMR are given by

$$
n_{0}=\left(3 \pi^{2}\right)^{-1}\left(\frac{2 m_{c}}{\hbar^{2}}\right)^{3 / 2}\left[M_{5}\left(E_{F B 0}\right)+N_{5}\left(E_{F B 0}\right)\right],
$$

and

$$
\frac{D}{\mu}=\frac{1}{e} \frac{\left[M_{5}\left(E_{F B 0}\right)+N_{5}\left(E_{F B 0}\right)\right]}{\left[M_{5}^{\prime}\left(E_{F B 0}\right)+N_{5}^{\prime}\left(E_{F B 0}\right)\right]},
$$

where $\quad M_{5}\left(E_{F B 0}\right) \equiv\left[E_{F B 0}\left(1+a E_{F B 0}\right)\right]^{3 / 2}$ and $N_{5}\left(E_{F B 0}\right)$ $\equiv \sum_{r=1}^{s_{0}} Z_{r} M_{5}\left(E_{F B 0}\right)$.

(iii) Under the constraints $\Delta \gg a^{-1}$ or $\Delta \ll a^{-1}$ together with the condition of $a E_{F B 0} \ll 1$, Eqs. (87) and (88) assume the forms

$$
n_{0}=N_{c}\left[F_{1 / 2}(\eta)+\left(\frac{15 \alpha k_{B} T}{4}\right) F_{3 / 2}(\eta)\right]
$$

and

$$
\frac{D}{\mu}=\left[\frac{k_{B} T}{e}\right]\left\{\frac{\left[F_{1 / 2}(\eta)+\left(\frac{15 \alpha k_{B} T}{4}\right) F_{3 / 2}(\eta)\right]}{\left[F_{-1 / 2}(\eta)+\left(\frac{15 \alpha k_{B} T}{4}\right) F_{1 / 2}(\eta)\right]}\right\},
$$

where $N_{C}=2\left(2 \pi m_{c} k_{B} T / h^{2}\right)^{3 / 2}$ and $\eta=E_{F B 0} / k_{B} T$.

(iv) For relatively wide gap materials $a \rightarrow 0$ and from Eqs. (89) and (90), we can write

$$
n_{0}=N_{c} F_{1 / 2}(\eta)
$$

and

$$
\frac{D}{\mu}=\left(\frac{k_{B} T}{e}\right)\left[\frac{F_{1 / 2}(\eta)}{F_{-1 / 2}(\eta)}\right] .
$$

Under the condition of carrier nondegeneracy, Eq. (92) gets simplified into the classical equation of the DMR as given in the Introduction.

\section{F. Suggestion for the experimental determination of the Einstein relation in materials having arbitrary dispersion laws}

It is well-known that the thermoelectric power of the electrons in materials in the presence of a very large magnetic field is independent of the scattering mechanism and is determined only by the dispersion law. ${ }^{80}$ The magnitude of the thermoelectric power $G$ in the present case can be expressed as ${ }^{80}$

$$
G=\frac{1}{e T N}\left[\int_{0}^{\infty}\left(E-\bar{E}_{F}\right) R(E)\left(-\frac{\partial f_{0}}{\partial E}\right) d E\right],
$$

where $R(E)$ is the total number of states and $f_{o}$ is the FermiDirac occupation probability factor. By following the work of Tsidilkovski, ${ }^{81}$ Eq. (93) can be written under the condition of carrier degeneracy as

$$
G=\left(\frac{\pi^{2} k_{B}^{2} T}{3 e N}\right)\left(\frac{\partial N}{\partial \bar{E}_{F}}\right) .
$$

The use of Eqs. (94) and (45) leads to the result

$$
\frac{D}{\mu}=\left(\pi^{2} k_{B}^{2} T / 3 G e^{2}\right) .
$$

Thus, the DMR for degenerate materials can be determined by knowing the experimental values of $G$.

The suggestion for the experimental determination of the DMR for degenerate materials having arbitrary dispersion laws as given by Eq. (95) does not contain any band parameters. For a constant temperature, the DMR inversely varies as $G$. Only the experimental values of $G$ for any material as a function of electron concentration will generate the experimental values of the DMR for that range of $N$ for that material. Since $G$ decreases with increasing $N$, from Eq. (95), we can conclude that the DMR will increase with increasing $N$. This statement provides a compatibility test of our theoretical analysis. Equation (95) provides an experimental check of the DMR and also a technique for probing the band structures of materials having arbitrary band structures.

\section{G. Six applications of the results of this paper in the field of quantum effect devices in general}

(i) The carrier contribution to the elastic constants is very important in studying the mechanical properties of the nanomaterials and has been relatively been less investigated in the literature. ${ }^{82}$ The electronic contribution to the second- and third-order elastic constants can be written as ${ }^{82,83}$

$$
\Delta C_{44}=-\frac{\alpha_{0}^{2}}{9} \frac{\partial N}{\partial \bar{E}_{F}}
$$

and 


$$
\Delta C_{456}=\frac{\alpha_{0}^{3}}{27} \frac{\partial^{2} N}{\partial \bar{E}_{F}^{2}},
$$

where $\alpha_{0}$ is the deformation potential constant. Thus, by using Eqs. (94), (96), and (97), we can write

$$
\Delta C_{44}=\left[-N \alpha_{0}^{2} e G /\left(3 \pi^{2} k_{B}^{2} T\right)\right]
$$

and

$$
\Delta C_{456}=\left(N e \alpha_{0}^{3} G^{2} /\left(3 \pi^{4} k_{B}^{3} T\right)\right)\left(1+\frac{N}{G} \frac{\partial G}{\partial N}\right) .
$$

Thus, the experimental graph of $G$ versus $N$ allows us to experimentally determine the electronic contribution to the elastic constants for nanomaterials having arbitrary dispersion laws.

(ii) The effective mass of the carriers in materials, being connected with the mobility, is known to be one of the most important quantity used for the analysis of the nanodevices under different physical conditions. ${ }^{84}$ It must be noted that among the various definitions of the effective electron mass, ${ }^{85}$ it is the momentum effective mass that should be regarded as the basic quantity. ${ }^{86}$ This is due to the fact that it is this mass which appears in the description of transport phenomena and all other properties of the conduction electrons in a band with arbitrary band nonparabolicity. ${ }^{86}$ It can be shown that it is the momentum effective mass which enters in various transport coefficients and plays the most dominant role in explaining the experimental results of different scattering mechanisms. ${ }^{87,88}$ The carrier degeneracy in semiconductors influences the effective mass when it is energy dependent. ${ }^{89}$ Under degenerate conditions, only the electrons at the Fermi surface of $n$-type semiconductors participate in the conduction process and, hence, the effective momentum mass (EMM) of the electrons corresponding to the Fermi level would be of interest in electron transport under such conditions. The Fermi energy is again determined by the carrier energy spectrum and the carrier concentration and, therefore, these two features would determine the dependence of the EMM in degenerate materials under the degree of carrier degeneracy. The expression of the EMM in the $i$ th direction is given by

$$
m_{i}^{*}\left(E_{F}\right)=\left.\hbar^{2}\left[k_{i_{0}}\left(\frac{\partial k_{i_{0}}}{\partial E}\right)\right]\right|_{E=E_{F}},
$$

where $i_{o}=x, y$, and $z$. Thus, by combining Eq. (100) with Eqs. (41)-(43), (46), (51), (56), (61), (66), and (73), we can obtain the expressions of the EMMs in QWs of III-V, ternary, and quaternary materials both in the presence and absence of photoexcitation. The detailed investigation of the EMMs will uncover new physics in this context.

(iii) The photoemission from nanostructures has been extensively investigated due to its importance in revealing many optical properties in the realm of nanoscience and technology, ${ }^{90}$ although the photoemission from the 1D systems has yet to be investigated. The generalized expression of the photoemitted current from the 1D structures as discussed in this paper can be written as

$$
I=\frac{e k_{B} T}{\pi \hbar} \sum_{n_{y}=1}^{n_{y_{\max }}} \sum_{n_{z}=1}^{n_{z_{\max }}}\left\{F_{0}\left[\frac{E_{F}-\left(E_{n}+W-h \nu\right)}{k_{B} T}\right]\right\},
$$

where $W$ is the work function and $h \nu$ is the incident photon energy along the direction of the free motion of the electron. From Eq. (101), we can observe that this generalized relation is valid for any $1 \mathrm{D}$ system where $E_{n}$ is the only band structure dependent quantity.

(iv) The normalized diffusion coefficient of the minority carriers in quantum wire lasers can be expressed ${ }^{4}$ as

$$
D_{i} / D_{0}=d E_{F} / d E_{F B 0},
$$

where $D_{i}$ and $D_{0}$ are the diffusion coefficients of the minority carriers both in the presence and absence of quantum confinements. Thus, our present study plays an important role in determining the diffusion coefficients of the minority carriers of quantum confined lasers with materials having arbitrary band structures.

(v) The nonlinear response from the optical excitation of the free carriers $\left(\bar{p}_{0}\right)$ is given by ${ }^{91}$

$$
\bar{p}_{0}=\frac{-e^{2}}{d_{y} d_{z} \omega^{2} \hbar^{2}} \int_{E_{n}}^{\infty}\left(k_{x} \frac{\partial k_{x}}{\partial E}\right)^{-1} f_{0} N_{1 D}(E) d E
$$

From the band structure, we can formulate the term $\left(k_{x} \partial k_{x} / \partial E\right)$, and by using the DOS function as formulated, we can study $\bar{p}_{0}$ for all types of materials as considered in this paper.

(vi) The third-order nonlinear optical susceptibility can be written as ${ }^{92}$

$$
\chi_{N P}\left(\omega_{1}, \omega_{2}, \omega_{3}\right)=\frac{n_{1 D} e^{4}\left\langle\varepsilon^{4}\right\rangle}{24 \omega_{1} \omega_{2} \omega_{3} d_{y} d_{z}\left(\omega_{1}+\omega_{2}+\omega_{3}\right) \hbar^{4}},
$$

where $n_{1 D}\left\langle\varepsilon^{4}\right\rangle=\int_{E_{n}}^{\infty}\left(\partial^{4} E / \partial k_{x}^{4}\right) N_{1 D}(E) f_{0} d E$ and the other notations are defined in Ref. 84 . The term $\left(\partial^{4} E / \partial k_{x}^{4}\right)$ can be formulated by using the band structure of different materials as given in this paper together with the fact that the respective DOS function has also been derived. Therefore, we can study the $\chi_{N P}\left(\omega_{1}, \omega_{2}, \omega_{3}\right)$ for all materials as considered in this paper.

Thus, we can summarize the whole theoretical background in the following way. In this paper, we have investigated the 1D DMR in QWs of III-V, ternary, and quaternary materials in the presence of photoexcitation on the basis of a newly formulated electron dispersion laws whose unperturbed conduction electrons obey the three band model of Kane. Under certain special conditions, we have also obtained the special results for materials whose unperturbed electron energy spectra are defined by the two band model of Kane and that of the parabolic energy bands. We have also investigated the DMR both in the presence and absence of photoexcitation in bulk specimens of the aforementioned materials. The well-known classical equation of the DMR has been obtained as special cases of our generalized analysis under certain constraints. ${ }^{22}$ This indirect test not only exhib- 
TABLE I. Values of the energy band constants.

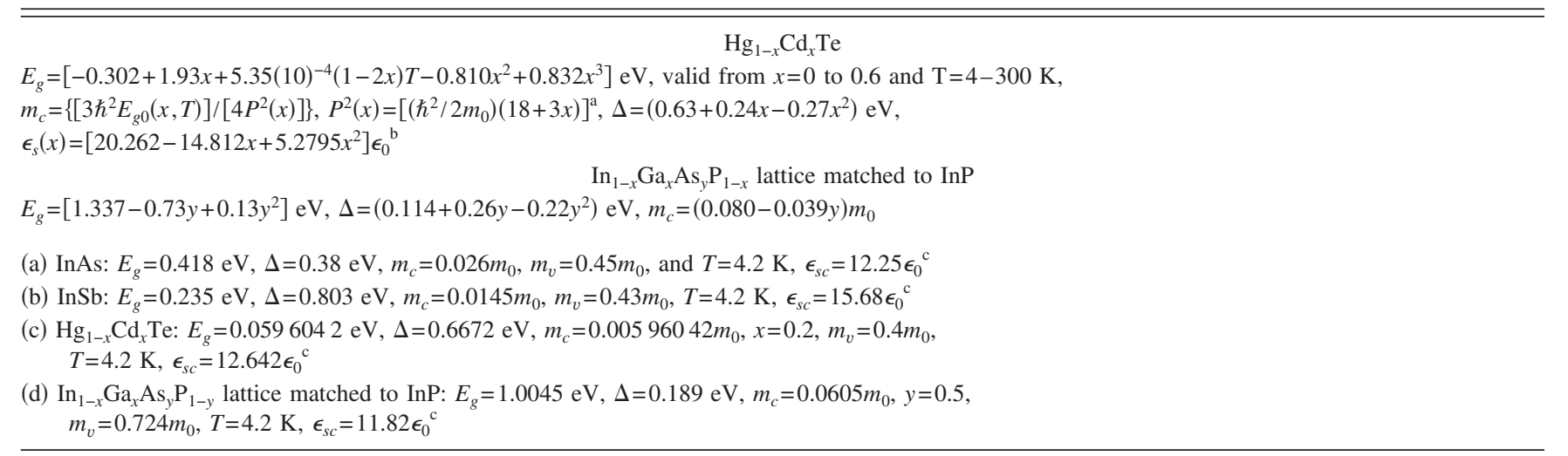

${ }^{\mathrm{a}}$ Reference 35.

${ }^{\mathrm{b}}$ Reference 36.

${ }^{c}$ Reference 93.

its the compatibility of our formulation but also shows the fact that our simple analysis is a more generalized one, since one can obtain the corresponding results for relatively wide gap three-dimensional materials having parabolic energy bands under certain constraints from our present derivation. We have suggested an experimental method for the determination of DMR, for nanomaterials having arbitrary dispersion laws. Besides, the results of this paper find six specific applications in the realm of nanodevices.

\section{RESULT AND DISCUSSIONS}

Taking the values of the energy band constants as given in Table I and using Eqs. (49), (50), (54), (55), (59), and (60), in Figs. 1-4, we have plotted the normalized 1D DMR as a function of the film thickness for QWs of $n$-InAs, ${ }^{93} n$-InSb, ${ }^{93}$ $\mathrm{Hg}_{1-x} \mathrm{Cd}_{x} \mathrm{Te},{ }^{93}$ and $\mathrm{In}_{1-x} \mathrm{Ga}_{x} \mathrm{As}_{y} \mathrm{P}_{1-y}$ lattice matched to InP (Ref. 93) in the presence [plots (a), (b), and (c)] and in the absence of photoexcitation [plots (d), (e), and (f)], where the said curves chronologically represent the three band model of Kane, two band model of Kane, and that of the parabolic energy bands, respectively. In Figs. 5-16, we have plotted the normalized 1D DMR as a function of electron concentra- tion per unit length, light intensity, and wavelength in the visible region, respectively, for all cases of Figs. 1-4. In Figs. 17 and 18, we have plotted the normalized 1D DMR as a function of alloy composition for $\mathrm{Hg}_{1-x} \mathrm{Cd}_{x} \mathrm{Te}$ and $\mathrm{In}_{1-x} \mathrm{Ga}_{x} \mathrm{As}_{y} \mathrm{P}_{1-y}$ lattice matched to InP, respectively, in the present case.

From Figs. 1-8, we observe that the 1D DMR decreases from the light off case to the light on case, since the value of the mobility in the presence of light waves becomes larger due to the increase in the electron velocity in the presence of light as compared to the same in the absence of photoexcitation. Therefore, the numerical magnitude of the 1D DMR in the presence of light is smaller as compared to the same in the light off case in the whole range of the film thickness and electron concentration per unit length as considered, although the 1D DMR decreases with increasing film thickness and increases with increase in carrier degeneracy.

The combined influences of the energy band constants on the 1D DMR for all the said compounds can easily be assessed from all the figures. For the purpose of relative assessment, all the plots in the absence of light waves have further been drawn. In Figs. 9-12, we observe that the 1D DMR decreases with increasing light intensity, whereas in

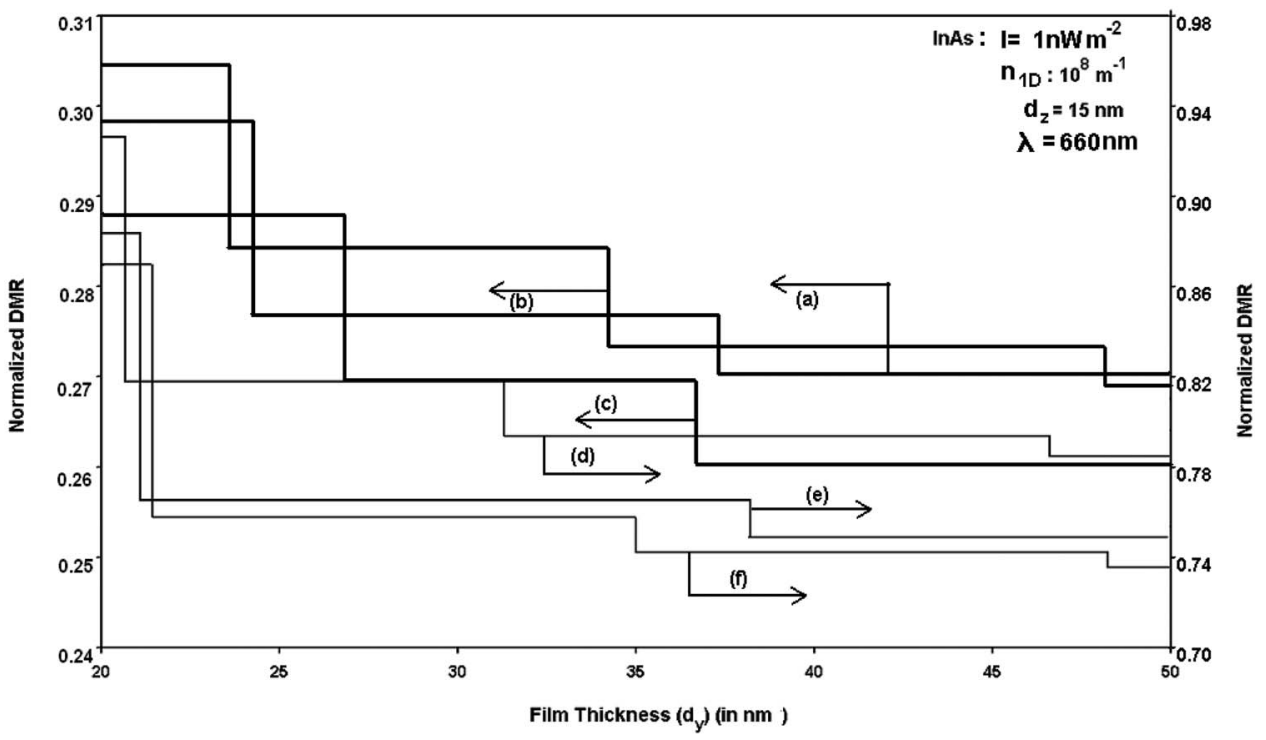

FIG. 1. Plot of the normalized 1D DMR as a function of film thickness for QWs of $n$-InAs in the presence of light waves in which the curves (a), (b), and (c) represent the three and two band models of Kane and that of the parabolic energy bands, respectively. The curves (d), (e), and (f) represent the same in the absence of external photoexcitation. 

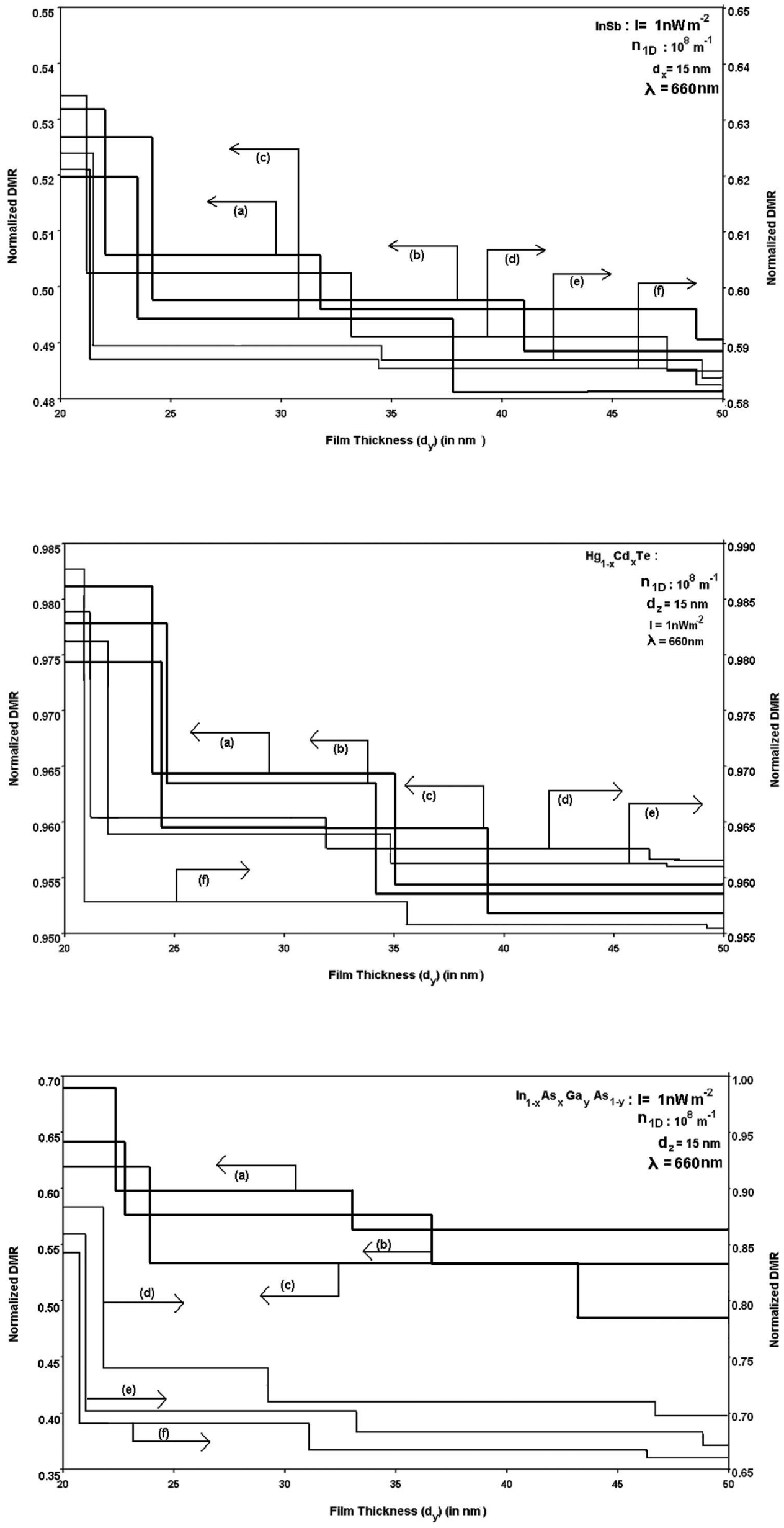

FIG. 2. Plot of the normalized 1D DMR as a function of film thickness for QWs of $n$-InSb in the presence of light waves in which the curves (a), (b), and (c) represent the three and two band models of Kane and that of the parabolic energy bands, respectively. The curves (d), (e), and (f) represent the same in the absence of external photoexcitation.
FIG. 3. Plot of the normalized 1D DMR as a function of film thickness for QWs of $n-\mathrm{Hg}_{1-x} \mathrm{Cd}_{x} \mathrm{Te}$ in the presence of light waves in which the curves (a), (b), and (c) represent the three and two band models of Kane and that of the parabolic energy bands, respectively. The curves (d), (e), and (f) represent the same in the absence of external photoexcitation.
FIG. 4. Plot of the normalized 1D DMR as a function of film thickness for QWs of $\mathrm{In}_{1-x} \mathrm{Ga}_{x} \mathrm{As}_{y} \mathrm{P}_{1-y}$ lattice matched to InP in the presence of light waves in which the curves (a), (b), and (c) represent the three and two band models of Kane and that of the parabolic energy bands, respectively. The curves (d), (e), and (f) represent the same in the absence of external photoexcitation. 

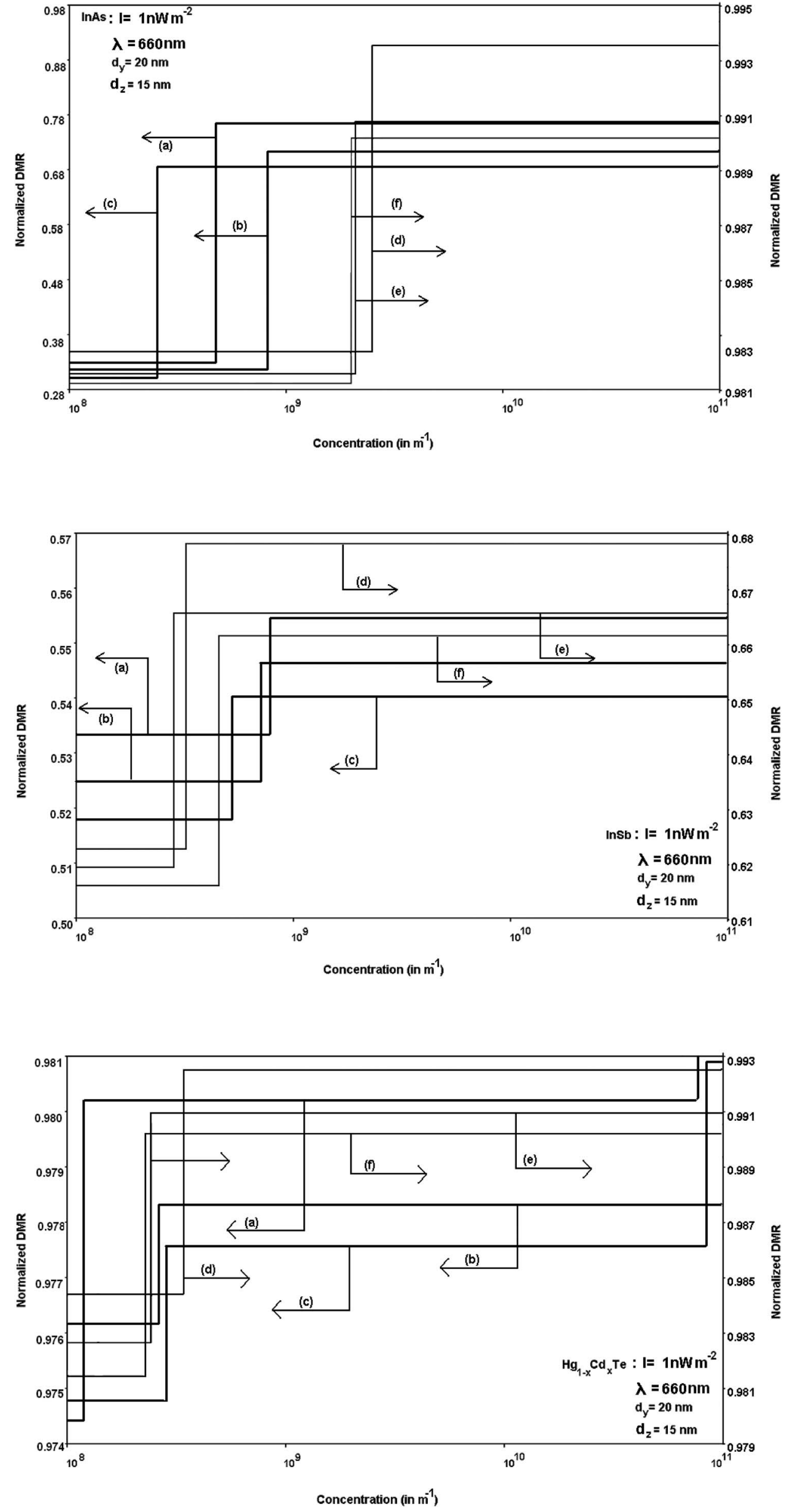

FIG. 5. Plot of the normalized 1D DMR as a function of electron concentration per unit length for QWs of $n$-InAs in the presence of light waves in which the curves (a), (b), and (c) represent the three and two band models of Kane and that of the parabolic energy bands, respectively. The curves (d), (e), and (f) represent the same in the absence of external photoexcitation.
FIG. 6. Plot of the normalized 1D DMR as a function of electron concentration per unit length for QWs of $n$-InSb in the presence of light waves in which the curves (a), (b), and (c) represent the three and two band models of Kane and that of the parabolic energy bands, respectively. The curves (d), (e), and (f) represent the same in the absence of external photoexcitation.
FIG. 7. Plot of the normalized 1D DMR as a function of electron concentration per unit length for QWs of $n-\mathrm{Hg}_{1-x} \mathrm{Cd}_{x} \mathrm{Te}$ in the presence of light waves in which the curves (a), (b), and (c) represent the three and two band models of Kane and that of the parabolic energy bands, respectively. The curves (d), (e), and (f) represent the same in the absence of external photoexcitation. 

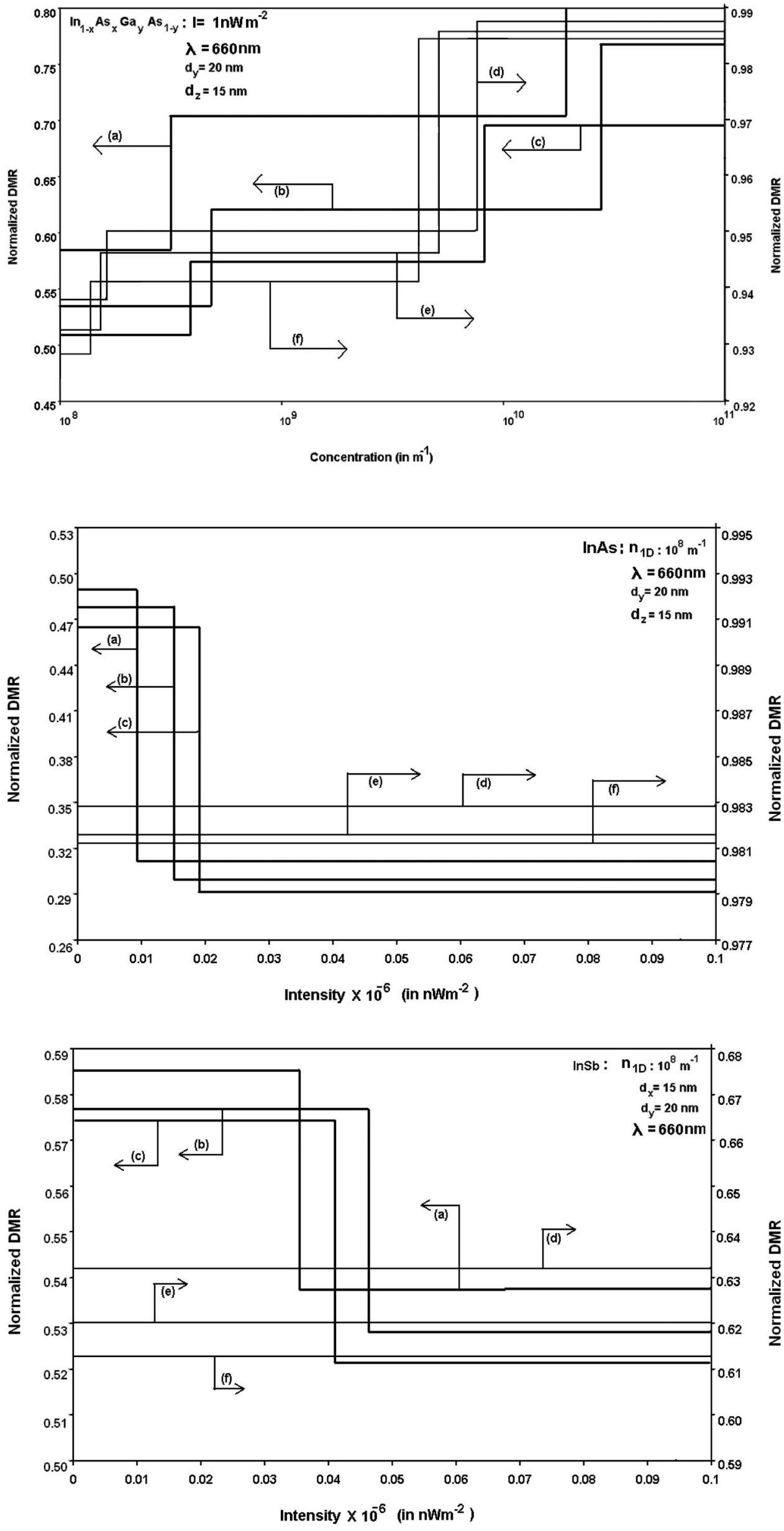

FIG. 8. Plot of the normalized 1D DMR as a function of electron concentration per unit length for QWs of $\mathrm{In}_{1-x} \mathrm{Ga}_{x} \mathrm{As}_{y} \mathrm{P}_{1-y}$ lattice matched to InP in the presence of light waves in which the curves (a), (b), and (c) represent the three and two band models of Kane and that of the parabolic energy bands, respectively. The curves (d), (e), and (f) represent the same in the absence of external photoexcitation.

FIG. 9. Plot of the normalized 1D DMR as a function of light intensity for QWs of $n$-InAs in which the curves (a), (b), and (c) represent the three and two band models of Kane and that of parabolic energy bands, respectively. The curves (d), (e), and (f) represent the same in the absence of external photoexcitation.

FIG. 10. Plot of the normalized 1D DMR as a function of light intensity for QWs of $n$-InSb in which the curves (a), (b), and (c) represent the three and two band models of Kane and that of parabolic energy bands, respectively. The curves (d), (e), and (f) represent the same in the absence of external photoexcitation. 


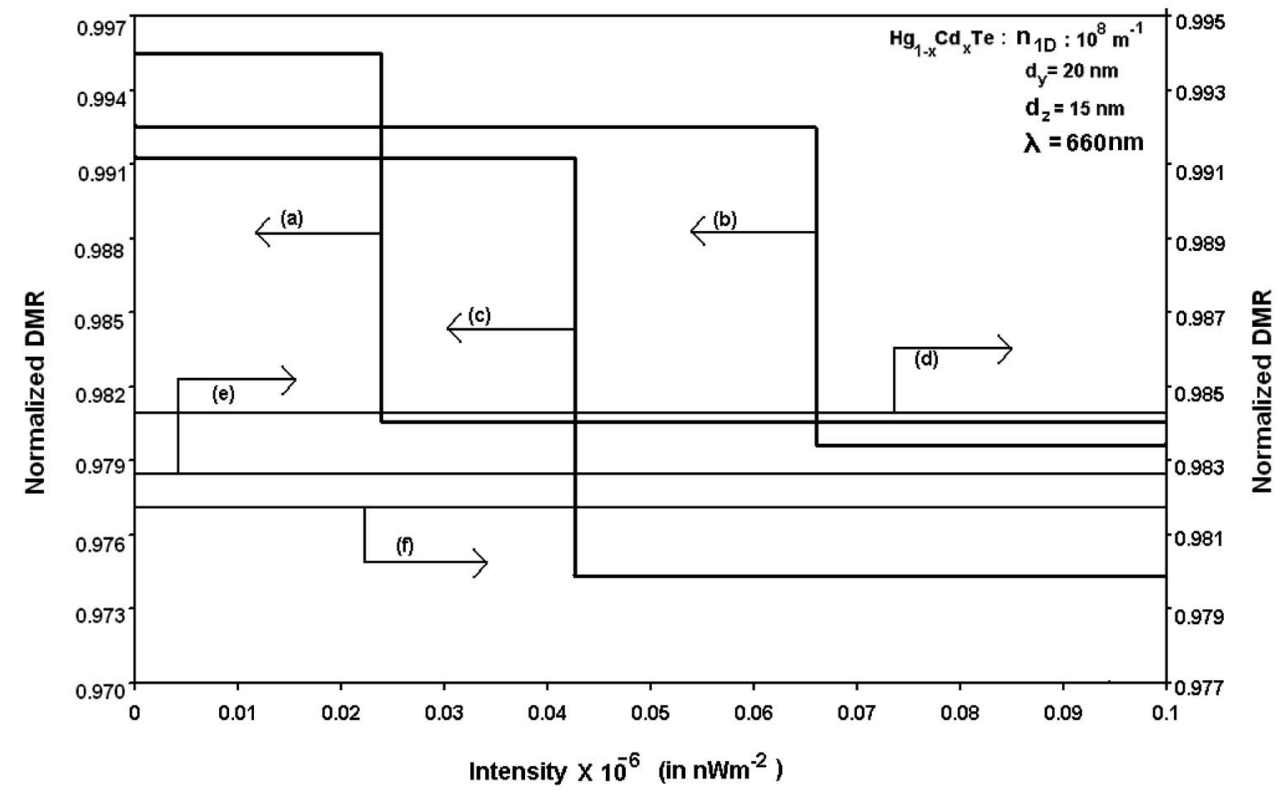

FIG. 11. Plot of the normalized 1D DMR as a function of light intensity for QWs of $n-\mathrm{Hg}_{1-x} \mathrm{Cd}_{x} \mathrm{Te}$ in which the curves (a), (b), and (c) represent the three and two band models of Kane and that of parabolic energy bands, respectively. The curves (d), (e), and (f) represent the same in the absence of external photoexcitation. the absence of external photoexcitation, the 1D DMR is independent of intensity. Figures 13-16 exhibit the fact that the 1D DMR decreases as the wavelength shifts from red color to violet. The plots of Fig. 17 are valid for $x>0.17$, since for $x<0.17$, the band gap becomes negative in $n-\mathrm{Hg}_{1-x} \mathrm{Cd}_{x} \mathrm{Te}$ leading to semimetallic state. The plots of Fig. 18 exhibit the variation of the $1 \mathrm{D}$ DMR with the alloy composition for $n$ - $\mathrm{In}_{1-x} \mathrm{Ga}_{x} \mathrm{As}_{y} \mathrm{P}_{1-y}$ lattice matched to InP.

The influence of quantum confinement on the aforementioned materials is immediately apparent from Figs. 1-4, since the 1D DMR strongly depends on the thickness of the size quantized materials, which is in direct contrast to their respective bulk specimens. It appears from the said figures that the 1D DMR decreases with the increasing film thickness in a steplike manner both in the presence and absence of photoexcitation for all types of materials as considered here, although the numerical values widely vary and determined by the constants of the energy spectra. The oscillatory depen- dence is due to the crossing over of the Fermi level by the size quantized levels. For each coincidence of a size quantized level with the Fermi level, there would be a discontinuity in the DOS function resulting in a peak of oscillations. With large values of film thickness, the height of the steps decreases and the 1D DMR will decrease with increasing film thickness in nonoscillatory manner and exhibit monotonic decreasing dependence. The height of step size and the rate of decrement are totally dependent on the band structure.

The influence of light is immediately apparent from the plots in Figs. 5-16, since the 1D DMR of QWs of the aforementioned compounds depends strongly on $I$ and $\lambda$ which is in direct contrast as compared to the corresponding cases of the same for their bulk specimens in the absence of external photoexcitation. The variations of the $1 D$ DMR in all the figures reflect the direct signature of the light waves on the electronic, optic, and the other band structure dependent properties of semiconducting materials in the presence of



FIG. 12. Plot of the normalized 1D DMR as a function of light intensity for QWs of $\mathrm{In}_{1-x} \mathrm{Ga}_{x} \mathrm{As}_{y} \mathrm{P}_{1-y}$ lattice matched to InP in which the curves (a), (b), and (c) represent the three and two band models of Kane and that of parabolic energy bands, respectively. The curves (d), (e), and (f) represent the same in the absence of external photoexcitation. 


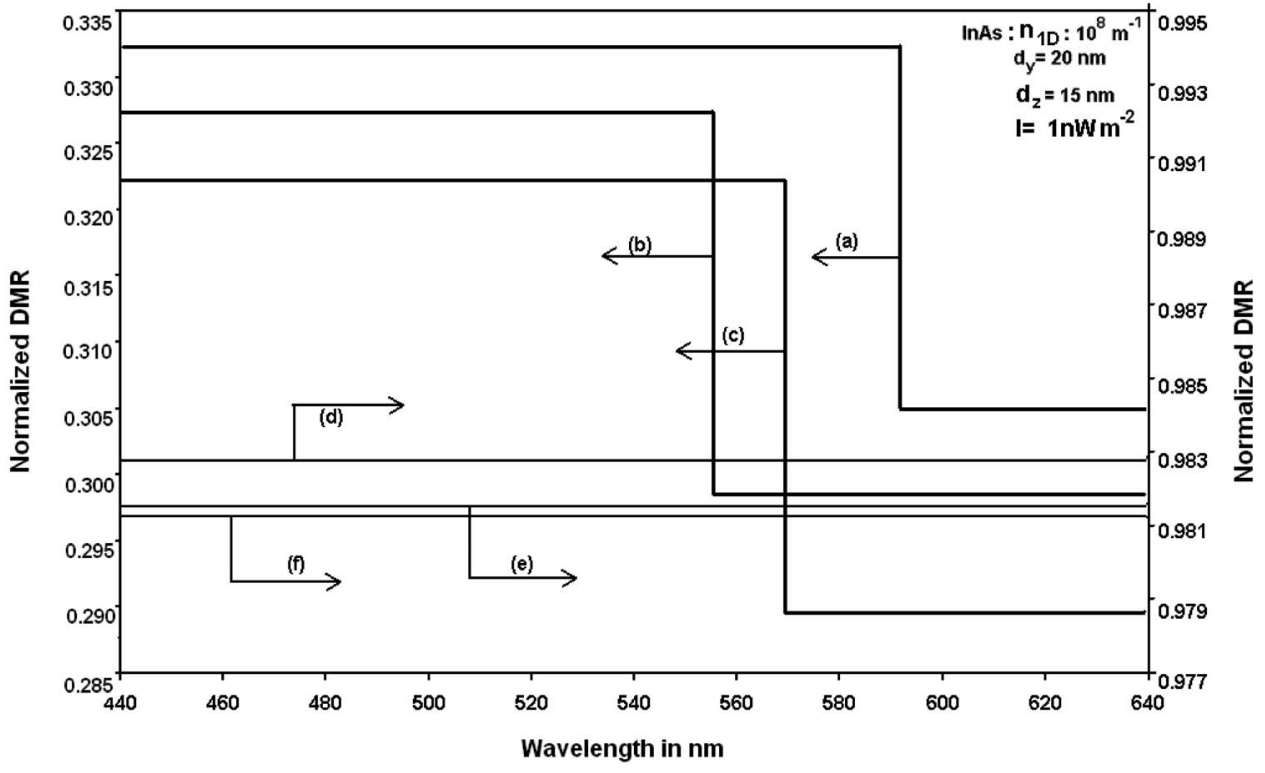

FIG. 13. Plot of the normalized 1D DMR as a function of wavelength for QWs of $n$-InAs in which the curves (a), (b), and (c) represent the three and two band models of Kane and that of parabolic energy bands respectively. The curves (d), (e), and (f) represent the same in the absence of external photoexcitation. light waves and the photon assisted transport for the corresponding semiconductor devices. Although the 1D DMR tends to decrease with the increasing intensity, wavelength, and decreasing electron concentration per unit length, the rate of variation is totally band structure dependent. It appears from the appropriate figures that the numerical values of the 1D DMR are greatest for ternary materials and least for quaternary compounds.

For both the ternary and quaternary materials, the 1D DMR continuously decreases with increasing alloy composition which is clearly apparent from Figs. 17 and 18, respectively. It appears from Figs. 5-8 that the 1D DMR increases with increasing carrier degeneracy which also reflects the $1 \mathrm{D}$ confinement through the steplike dependence. This oscillatory dependence will be less and less prominent with increasing film thickness and carrier concentration, respectively. Ultimately, for bulk specimens of the same material, the DMR will be found to continuously increase with increasing electron concentration in a nonoscillatory manner.
The appearance of the humps of the respective figures is due to the redistribution of the electrons among the quantized energy levels when the size quantum number corresponding to the highest occupied level changes from one fixed value to the others. With varying electron concentration, a change is reflected in the $1 \mathrm{D}$ DMR through the redistribution of the electrons among the size quantized levels. Our experimental suggestions for the determinations of the 1D DMR and, consequently, the carrier contribution to the elastic constants are valid for QWs having arbitrary dispersion relations. Since the experimental curves of $N$ versus $G$ are not available in literature for the present generalized systems, we cannot compare our theoretical formulation with the proposed experiment, although the generalized analysis as presented in this context can be checked when the experimental investigations of $G$ would appear in the literature.

In recent years, the mobility of electrons in QWs of nonparabolic semiconductors has been extensively investigated, but the diffusion constant (a very important device parameter

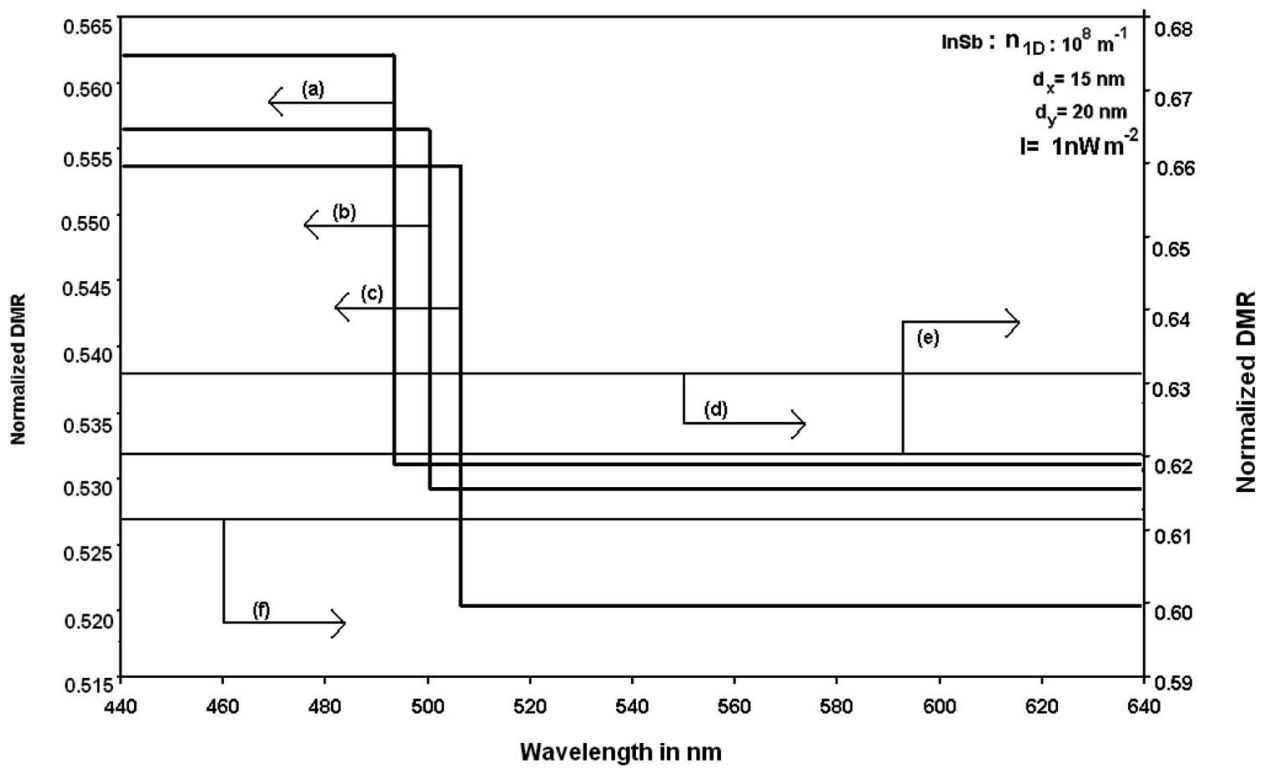

FIG. 14. Plot of the normalized 1D DMR as a function of wavelength for QWs of $n$-InSb in which the curves (a), (b), and (c) represent the three and two band models of Kane and that of parabolic energy bands, respectively. The curves (d), (e), and (f) represent the same in the absence of external photoexcitation. 


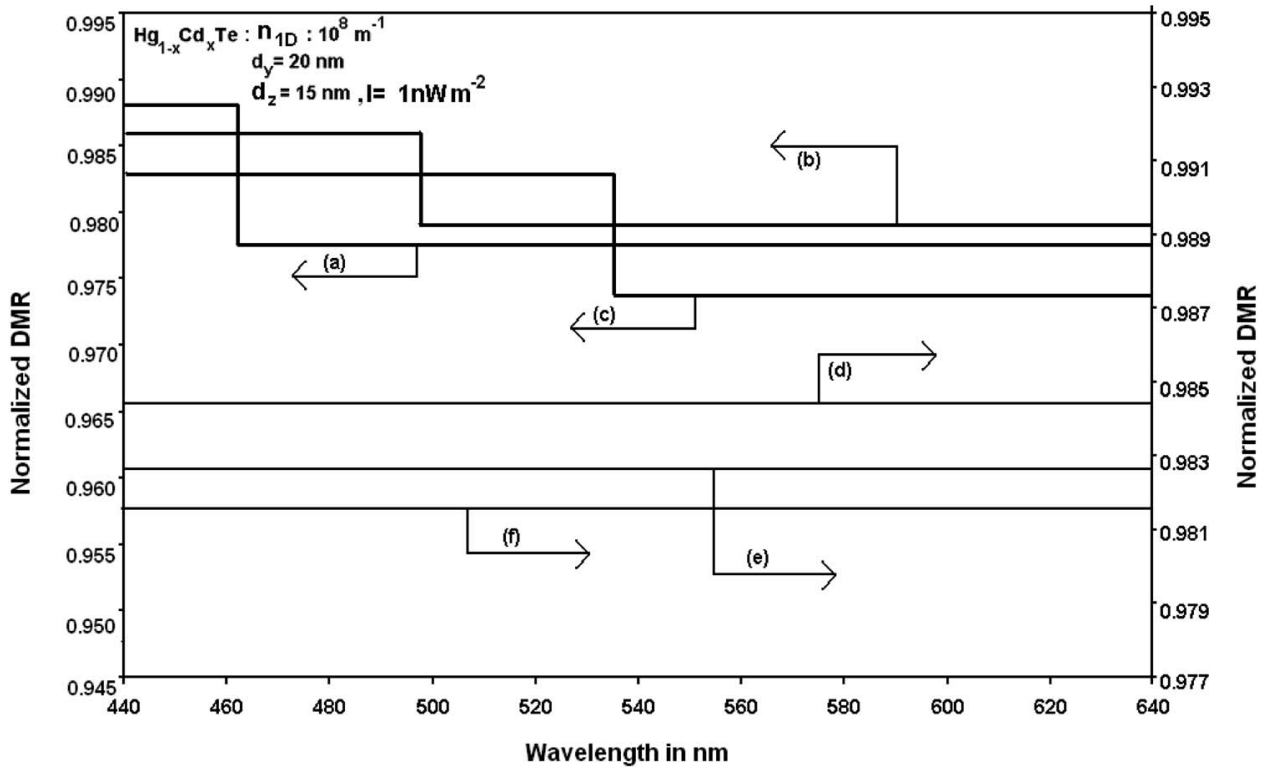

FIG. 15. Plot of the normalized 1D DMR as a function of wavelength for QWs of $n-\mathrm{Hg}_{1-x} \mathrm{Cd}_{x} \mathrm{Te}$ in which the curves (a), (b), and (c) represent the three and two band models of Kane and that of parabolic energy bands, respectively. The curves (d), (e), and (f) represent the same in the absence of external photoexcitation.

which cannot be easily experimentally determined) of such materials has been relatively less studied. Thus, the theoretical results of our paper will be useful in determining the diffusion constants even for relatively wide gap compounds whose energy band structures can be approximated by the parabolic energy bands, both in the presence and absence of light waves. It is worth remarking that our basic Eq. (41) covers various materials having different energy band structures. In this paper, the film thickness, electron concentration per unit length, light intensities and wavelength in the visible region dependences of the $1 \mathrm{D}$ DMR for $n$-InAs, $n$-InSb, $n-\mathrm{Hg}_{1-x} \mathrm{Cd}_{x} \mathrm{Te}$ and $n-\mathrm{In}_{1-x} \mathrm{Ga}_{x} \mathrm{As}_{y} \mathrm{P}_{1-y}$ lattice matched to InP, both for QWs and their corresponding bulk specimens, have been studied. Thus, we have covered a wide class of QWs of optoelectronic and allied compounds whose energy band structures are defined by the three and two band models of Kane both in the presence and absence of light waves. Under certain limiting conditions, the result of the 1D DMR in the absence of external photoexcitation leads to the well-known expression of the DMR for relatively degenerate wide band gap materials having parabolic energy bands as obtained by Landsberg. ${ }^{4}$ This indirect test not only exhibits the mathematical compatibility of our formulation but also shows the fact that our simple analysis is a more generalized one.

We can conclude that the influence of the presence of an external photoexcitation is to radically change the original band structure of the material. Our method is not at all related to the DOS technique as used in the literature. ${ }^{94}$ From the Ek dispersion relation, we can obtain the DOS, but the DOS technique as used in literature ${ }^{94}$ cannot provide the carrier energy spectrum. Therefore, our study is more fundamental than those of the existing literature because the Boltzman transport equation, which controls the study of the charge transport properties of semiconductor devices, can be solved if and only if the Ek dispersion relation is known. We have not considered the hot electron and many body effects in this simplified theoretical formalism due to the lack of availability in literature of proper analytical techniques for

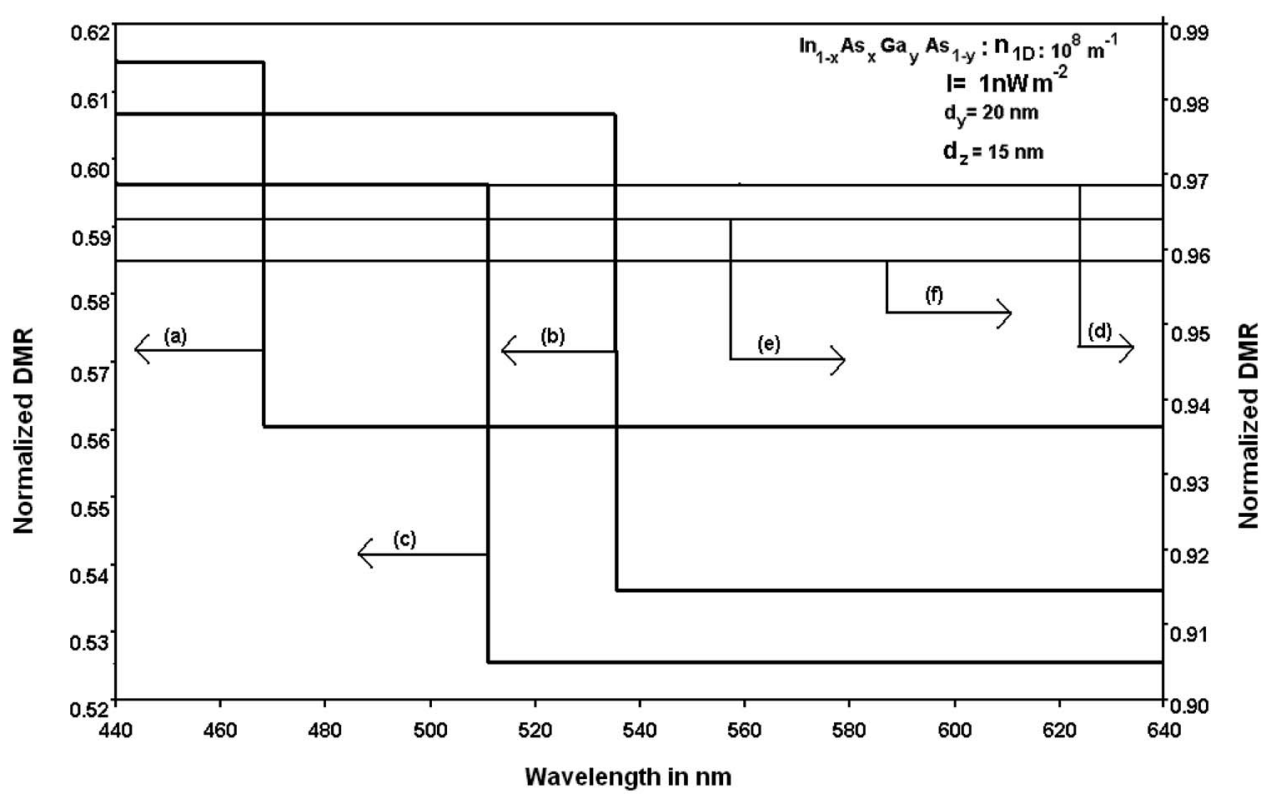

FIG. 16. Plot of the normalized 1D DMR as a function of wavelength for QWs of $\operatorname{In}_{1-x} \mathrm{Ga}_{x} \mathrm{As}_{y} \mathrm{P}_{1-y}$ lattice matched to $\mathrm{InP}$ in which the curves (a), (b), and (c) represent the three and two band models of Kane and that of parabolic energy bands, respectively. The curves (d), (e), and (f) represent the same in the absence of external photoexcitation. 


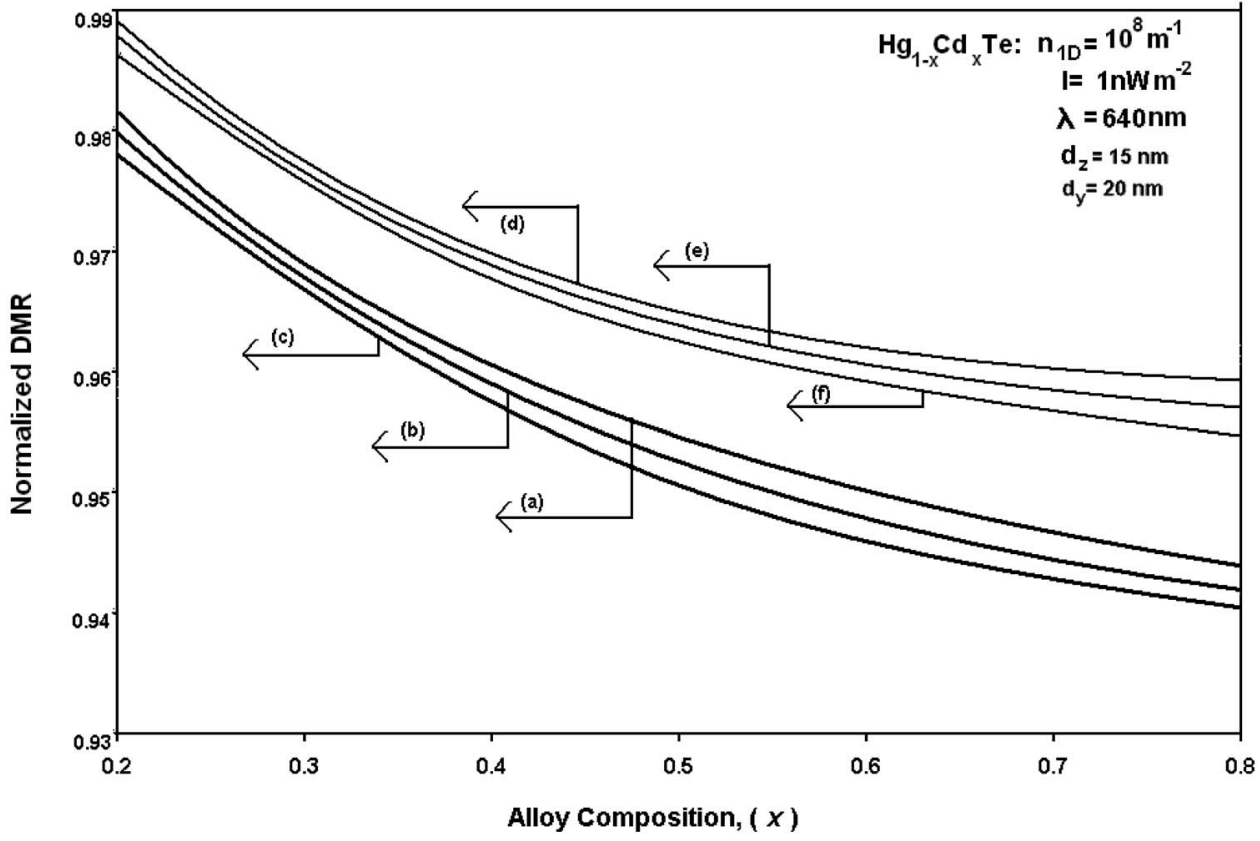

FIG. 17. Plot of the normalized 1D DMR as a function of alloy composition for QWs of $\mathrm{Hg}_{1-x} \mathrm{Cd}_{x} \mathrm{Te}$ in the presence of light waves in which the curves (a), (b), and (c) represent the three and two band models of Kane and that of parabolic energy band, respectively. The curves (d), (e), and (f) represent the same in the absence of external photoexcitation. including them for the generalized systems as considered in this paper. Our simplified approach will be useful for the purpose of comparison when methods of tackling the formidable problem of many body and the hot electron effects for the present generalized systems appear. The inclusion of the said effects would certainly increase the accuracy of the results, although the qualitative features of the DMR as discussed in this paper would not change in the presence of the aforementioned effects. Besides, our suggestion for the experimental determination of the $1 \mathrm{D}$ DMR is independent of the inclusion of the said effects. We have not considered other types of optoelectronic materials and other external variables to keep the presentation brief. The influence of energy band models and the various band constants on the 1D DMR for different materials can also be studied from all the figures of this paper.

It may be noted that the Einstein relation is a conse- quence of Boltzmann transport equation (BTE). ${ }^{21}$ The BTE for QWs and wires is valid when the nanometer dimensions $d_{x}$ or $d_{y}$ are of the order of de Broglie wavelength $\lambda_{d}$ and is not valid if the nanometer dimensions are much less than $\lambda_{d}{ }^{95}$ The order of $\lambda_{d}$ is about $10 \mathrm{~nm}$ in semiconductors. ${ }^{96}$ Thus, our formulation is valid for $\lambda_{d} \geqslant 10 \mathrm{~nm}$. For the purpose of numerical computations, as shown in various graphs, we have taken $d_{x}, d_{y}>10 \mathrm{~nm}$ in this context.

The numerical results presented in this paper would be different for other materials but the nature of variation would be unaltered. The theoretical results as given here would be useful in analyzing various other experimental data related to this phenomenon. Finally, we can write that the present analysis can be used to investigate the Burstien Moss shift, the plasma frequency, various scattering mechanisms, the specific heat, and other different transport coefficients of

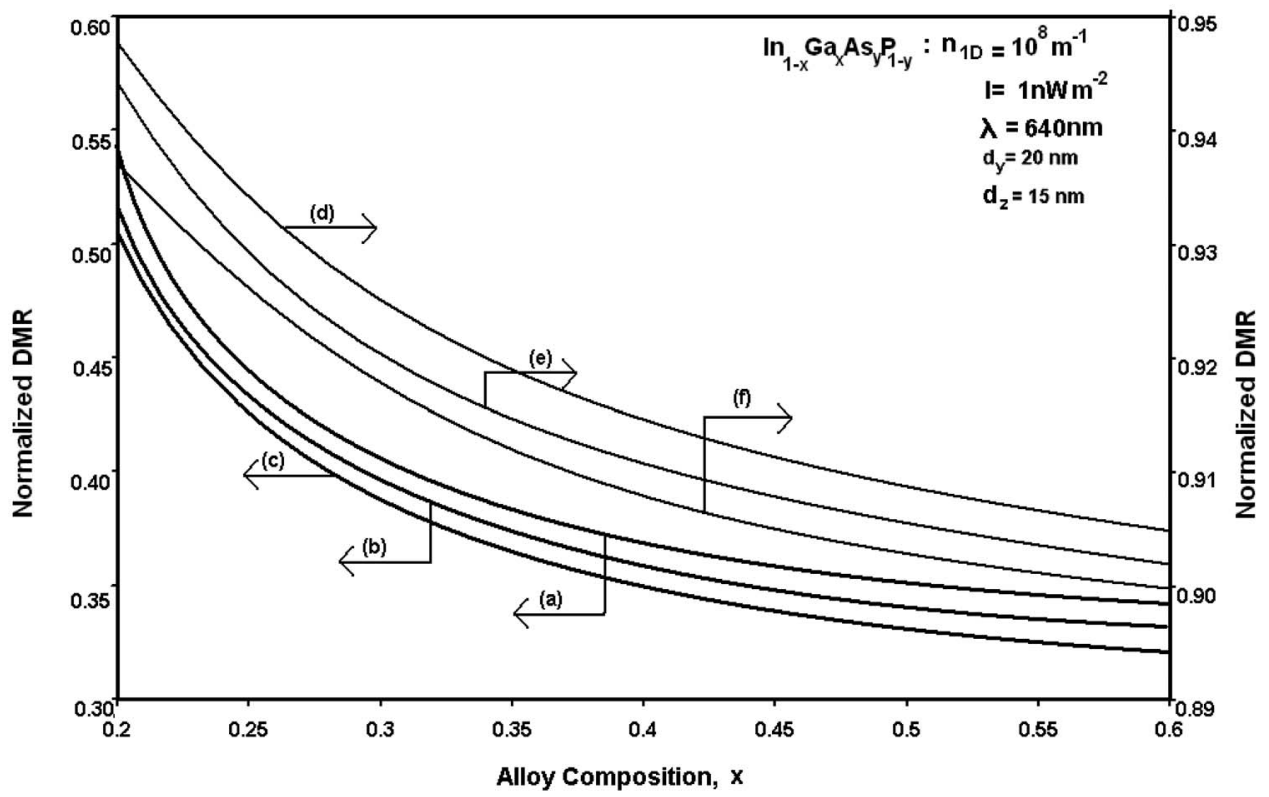

FIG. 18. Plot of the normalized 1D DMR as a function of alloy composition for QWs of $\mathrm{Hg}_{1-x} \mathrm{Cd}_{x} \mathrm{Te}$ in the presence of light waves in which the curves (a), (b), and (c) represent the three and two band models of Kane and that of parabolic energy band, respectively. The curves (d), (e), and (f) represent the same in the absence of external photoexcitation. 
modern quantum effect devices operated under the influence of external photon field.

\section{ACKNOWLEDGMENTS}

One of the authors, K. P. Ghatak, is grateful to All India Council of Technical Education for granting a project having Ref. No. RPS2008/G-3/126 under Research Promotion Scheme 2008.

${ }^{1}$ H. Kroemer, IEEE Trans. Electron Devices 25, 850 (1978).

${ }^{2}$ R. W. Lade, Proc. IEEE 51, 743 (1964).

${ }^{3}$ G. D. Hatchel and A. E. Ruchli, IEEE Trans. Electron Devices 15, 437 (1968); S. N. Mohammed, J. Phys. C 13, 2685 (1980).

${ }^{4}$ P. T. Landsberg, Eur. J. Phys. 2, 213 (1981).

${ }^{5}$ P. T. Landsberg, Thermodynamics and Statistical Mechanics (Oxford University Press, Oxford, 1978); In Recombination in Compounds (Cambridge University Press, Cambridge, 1991).

${ }^{6}$ P. T. Landsberg, J. Appl. Phys. 56, 1696 (1984)

${ }^{7}$ P. T. Landsberg and S. A. Hope, Solid-State Electron. 20, 421 (1977).

${ }^{8}$ S. A. Hope, G. Feat, and P. T. Landsberg, J. Appl. Phys. 61, 4909 (1987).

${ }^{9}$ P. T. Landsberg, Proc. R. Soc. London, Ser. A 213, 226 (1952).

${ }^{10}$ J. P. Bouchaud and A. Georges, Phys. Rep. 195, 127 (1996).

${ }^{11}$ R. K. Jain, Phys. Status Solidi A 42, K221 (1977).

${ }^{12}$ A. H. Marshak, Solid-State Electron. 30, 1089 (1987).

${ }^{13}$ A. H. Marshak and K. M. V. Vliet, Proc. IEEE 72, 148 (1984).

${ }^{14}$ K. M. V. Vliet and A. Van der Zeil, Solid-State Electron. 20, 931 (1977).

${ }^{15}$ A. N. Chakravarti and B. R. Nag, Int. J. Electron. 37, 281 (1974).

${ }^{16}$ B. R. Nag and A. N. Chakravarti, Solid-State Electron. 18, 109 (1975).

${ }^{17}$ B. R. Nag, A. N. Chakravarti, and P. K. Basu, Phys. Status Solidi A 68, K75 (1981).

${ }^{18}$ B. R. Nag and A. N. Chakravarti, Phys. Status Solidi A 67, K113 (1981).

${ }^{19}$ A. N. Chakravarti and D. P. Parui, Phys. Lett. 40, 113 (1972); 43, 60 (1973).

${ }^{20}$ D. Mukherjee, A. N. Chakravarti, and B. R. Nag, Phys. Status Solidi A 26 K27 (1974).

${ }^{21}$ B. R. Nag, Electron Transport in Compound Semiconductors (SpringerVerlag, Berlin 1980)

${ }^{22}$ A. N. Chakravarti, K. P. Ghatak, A. Dhar, K. K. Ghosh, and S. Ghosh, Appl. Phys. A: Solids Surf. 26, 169 (1981).

${ }^{23}$ M. Mondal and K. P. Ghatak, Phys. Scr. 30, 217 (1984).

${ }^{24}$ M. Mondal and K. P. Ghatak, J. Magn. Magn. Mater. 62, 115 (1986); J. Phys. C 20, 1671 (1987); 46, 502 (1989).

${ }^{25}$ M. Mondal and K. P. Ghatak, Thin Solid Films 148, 219 (1987).

${ }^{26}$ K. P. Ghatak, A. K. Choudhury, S. Ghosh, and A. N. Chakravarti, Appl Phys. 23, 241 (1980)

${ }^{27}$ M. Mondal, N. Chattopadhyay, and K. P. Ghatak, J. Low Temp. Phys. 73 321 (1988).

${ }^{28}$ K. P. Ghatak, N. Chattopadhyay, and M. Mondal, J. Appl. Phys. 44, 305 (1987); 63, 4536 (1988); K. P. Ghatak, B. Mitra, and M. Mondal, Ann. Phys. 48, 283 (1990); K. P. Ghatak and S. N. Biswas, J. Appl. Phys. 70, 4309 (1991).

${ }^{29}$ B. Mitra and K. P. Ghatak, Phys. Lett. A 135, 397 (1989).

${ }^{30}$ B. Mitra and K. P. Ghatak, Solid-State Electron. 32, 810 (1989).

${ }^{31}$ S. N. Biswas and K. P. Ghatak, Int. J. Electron. 73, 287 (1992).

${ }^{32}$ K. P. Ghatak, "Influence of Band Structure on Some Quantum Processes of tetragonal semiconductors," D.Eng. thesis, Jadavpur University, 1991.

${ }^{33}$ K. P. Ghatak, B. Mitra, and B. Nag, Phys. Status Solidi B 199, 95 (1997).

${ }^{34}$ S. Choudhury, L. J. Singh, and K. P. Ghatak, Nanotechnology 15, 180 (2004).

${ }^{35}$ H. Sasaki, Jpn. J. Appl. Phys. 19, 94 (1980).

${ }^{36}$ P. M. Petroff, A. C. Gossard, R. A. Logan, and W. Weigmann, Appl. Phys. Lett. 41, 635 (1982).

${ }^{37}$ I. Miller, A. Miller, A. Shahar, U. Koren, and P. J. Corvini, Appl. Phys. Lett. 54, 188 (1989).

${ }^{38}$ F. Sols, M. Macucci, U. Ravaioli, and K. Hess, Appl. Phys. Lett. 54, 350 (1980).

${ }^{39}$ C. S. Lent and D. J. Kirkner, J. Appl. Phys. 67, 6353 (1990).

${ }^{40}$ C. S. Kim, A. M. Satanin, Y. S. Joe, and R. M. Cosby, Phys. Rev. B 60, 10962 (1999).

${ }^{41}$ S. Midgley and J. B. Wang, Phys. Rev. B 64, 153304 (2001).

${ }^{42}$ T. Sugaya, J. P. Bird, M. Ogura, Y. Sugiyama, D. K. Ferry, and K. Y. Jang, Appl. Phys. Lett. 80, 434 (2002).
${ }^{43}$ B. Kane, G. Facer, A. Dzurak, N. Lumpkin, R. Clark, L. PfeiKer, and K. West, Appl. Phys. Lett. 72, 3506 (1998).

${ }^{44}$ C. Dekker, Phys. Today 52(5), 22 (1999).

${ }^{45}$ A. Yacoby, H. L. Stormer, N. S. Wingreen, L. N. Pfeiffer, K. W. Baldwin, and K. W. West, Phys. Rev. Lett. 77, 4612 (1996).

${ }^{46}$ Y. Hayamizu, M. Yoshita, S. Watanabe, H. A. L. PfeiKer, and K. West, Appl. Phys. Lett. 81, 4937 (2002).

${ }^{47}$ S. Frank, P. Poncharal, Z. L. Wang, and W. A. Heer, Science 280, 1744 (1998).

${ }^{48}$ I. Kamiya, I. I. Tanaka, K. Tanaka, F. Yamada, Y. Shinozuka, and H. Sakaki, Physica E (Amsterdam) 13, 131 (2002).

${ }^{49}$ A. K. Geim, P. C. Main, N. La Scala, Jr., L. Eaves, T. J. Foster, P. H. Beton, J.-W. Sakai, F. W. Sheard, M. Henini, G. Hill, and M. A. Pate, Phys. Rev. Lett. 72, 2061 (1994).

${ }^{50}$ A. S. Melinkov and V. M. Vinokur, Nature (London) 415, 60 (2002).

${ }^{51}$ K. Schwab, E. A. Henriksen, J. M. Worlock, and M. L. Roukes, Nature (London) 404, 974 (2000)

${ }^{52}$ L. Kouwenhoven, Nature (London) 403, 374 (2000).

${ }^{53}$ S. Komiyama, O. Astafiev, V. Antonov, and H. Hirai, Nature (London) 403, 405 (2000).

${ }^{54}$ E. Paspalakis, Z. Kis, E. Voutsinas, and A. F. Terziz, Phys. Rev. B 69, 155316 (2004).

${ }^{55}$ J. H. Jefferson, M. Fearn, D. L. J. Tipton, and T. P. Spiller, Phys. Rev. A 66, 042328 (2002).

${ }^{56}$ J. Appenzeller, C. Schroer, T. Schapers, A. Hart, A. Froster, B. Lengeler, and H. Luth, Phys. Rev. B 53, 9959 (1996).

${ }^{57}$ J. Appenzeller and C. Schroer, J. Appl. Phys. 87, 3165 (2000).

${ }^{58}$ P. Debray, O. E. Raichev, M. Rahman, R. Akis, and W. C. Mitchel, Appl. Phys. Lett. 74, 768 (1999).

${ }^{59}$ P. M. Solomon, Proc. IEEE 70, 489 (1982); T. E. Schlesinger and T. Kuech, Appl. Phys. Lett. 49, 519 (1986).

${ }^{60}$ H. Heiblum, D. C. Thomas, C. M. Knoedler, and M. I. Nathan, Appl. Phys. Lett. 47, 1105 (1985).

${ }^{61}$ O. Aina, M. Mattingly, F. Y. Juan, and P. K. Bhattacharyya, Appl. Phys. Lett. 50, 43 (1987).

${ }^{62}$ I. Suemune and L. A. Coldren, IEEE J. Quantum Electron. 24, 1178 (1988).

${ }^{63}$ D. A. B. Miller, D. S. Chemla, T. C. Damen, J. H. Wood, A. C. Burrus, A. C. Gossard, and W. Weigmann, IEEE J. Quantum Electron. 21, 1462 (1985).

${ }^{64}$ R. Bisaro, G. Laurencin, A. Friederich, M. Razeghi, Appl. Phys. Lett. 40, 978 (1982).

${ }^{65}$ R. C. Alferness, C. H. Joyner, M. D. Divino, M. J. R. Martyak, and L. L. Buhl, Appl. Phys. Lett. 49, 125 (1986).

${ }^{66}$ T. E. Bell, IEEE Spectrum 20, 38 (1983).

${ }^{67}$ P. Y. Lu, C. H. Wang, C. M. Williams, S. N. G. Chu, and C. M. Stiles, Appl. Phys. Lett. 49, 1372 (1986).

${ }^{68}$ N. K. Taskar, I. B. Bhat, K. K. Parat, D. Terry, H. Ehsani, and S. K. Gandhi, J. Vac. Sci. Technol. A 7, 281 (1989).

${ }^{69}$ E. Weiss and N. Mainzer, J. Vac. Sci. Technol. A 7, 391 (1989).

${ }^{70}$ F. Koch, Two Dimensional Systems, Heterostructures and Superlattices, Springer Series in Solid State Science, edited by G. Bauer, F. Kuchar, and H. Heinrich, Vol. 53 (Springer-Verlag, Berlin 1984).

${ }^{71}$ D. H. Daw, D. S. Caoand, and G. B. Stringfellow, J. Appl. Phys. 69, 2552 (1991).

${ }^{72}$ M. L. Timmons, S. Bedair, M. R. J. Markunas, and J. A. Hutchby, Proceedings of 16th IEEE Photovoltaic Specialists Conferences (IEEE, San Diego, CA, 1982), Vol. 666

${ }^{73}$ M. K. Lee, D. S. Wu, and H. H. Tung, J. Appl. Phys. 62, 3209 (1987).

${ }^{74}$ G. L. Hansen, J. L. Schmit, and T. N. Casselman, J. Appl. Phys. 63, 7099 (1982).

${ }^{75}$ S. K. Sutradhar, D. Chattopadhyay, and B. R. Nag, Phys. Rev. B 25, 4069 (1982).

${ }^{76}$ K. Seeger, Semiconductor Physics, 7th ed. (Springer-Verlag, Berlin, 2006).

${ }^{77}$ B. R. Nag, Physics of Quantum Well Devices (Kluwer, Dordrecht, 2000).

${ }^{78}$ M. Abramowitz and I. A. Stegun, Handbook of Mathematical Functions (Dover, New York, 1965).

${ }^{79}$ B. Mitra and K. P. Ghatak, Phys. Scr. 42, 103 (1990).

${ }^{80} \mathrm{~W}$. Zawadzki, Two-Dimensional Systems, Hetrostructures and Superlattices, Springer Series in Solid-State Science Vol. 53, edited by G. Bower, F. Kuches, and H. Haiunich (Springer, Berlin, 1984), p. 79; K. P. Ghatak and M. Mondal, J. Appl. Phys. 66, 3056 (1989).

${ }^{81}$ I. M. Tsidilkovskii, Band Structures of Compounds (Pergamon, London, 1982); B. Mitra and K. P. Ghatak, Phys. Lett. A 141, 81 (1989). 
${ }^{82}$ A. K. Sreedhar and S. C. Gupta, Phys. Rev. B 5, 3160 (1972).

${ }^{83}$ K. P. Ghatak, J. P. Banerjee, M. Mitra, and B. Nag, Mol. Cryst. Liq. Cryst. Sci. Technol., Sect. B: Nonlinear Opt. 16, 193 (1996); B. Nag and K. P. Ghatak, J. Phys. Chem. Solids 58, 427 (1997).

${ }^{84}$ S. Adachi, J. Appl. Phys. 58, R11 (1985).

${ }^{85}$ R. Dornhaus and G. Nimtz, Springer Tracts in Modern Physics (Springer, Berlin, 1976), 78, p. 1.

${ }^{86}$ W. Zawadzki, Handbook of Semiconductor Physics, edited by W. Paul (North-Holland, Amsterdam, 1982), Vol. 1, p. 719.

${ }^{87}$ I. M. Tsidilkovski, Ph.D. thesis, Electronic Properties of Degenerate Semiconductors, Leningrad University, 1955.

${ }^{88}$ E. V. Rozenfeld, Yu. P. Trkhin, and P. G. Guletskii, Sov. Phys. Solid State 29, 1314 (1988)
${ }^{89}$ K. P. Ghatak and M. Mondal, Z. Naturforsch. Teil A 41A, 881 (1986); K. P. Ghatak and M. Mondal, Z. Phys. B: Condens. Matter 69, 471 (1988).

${ }^{90}$ K. P. Ghatak and M. Mondal, Solid-State Electron. 31, 1651 (1988).

${ }^{91}$ A. S. Filipchenko, I. G. Lang, D. N. Nasledov, S. T. Pavlov, and L. N. Radaikine, Phys. Status Solidi B 66, 417 (1974).

${ }^{92}$ M. Wegener, Extreme Nonlinear Optics (Springer-Verlag, Berlin, 2005).

${ }^{93}$ P. K. Chakraborty, S. Bhattacharya, and K. P. Ghatak, J. Appl. Phys. 98, 053517 (2005)

${ }^{94}$ J. N. Schulman and Y. C. Chang, Phys. Rev. B 24, 4445 (1981).

${ }^{95}$ V. V. Mitin, V. A. Kochelop, and M. A. Stroscio, Quantum Heterostructures (Cambridge University Press, Cambridge, 1999).

${ }^{96}$ S. Datta, Electron Transport in Mesoscopic Systems, (Cambridge University Press, Cambridge, 2001). 